\title{
MFTF Thomson scattering: a system study
}

\author{
A. M. Frank
}

Manuscript date: September 11, 1980



LAWRENCE LIVERMORE LABORATORY

University of California - Livermore, California • 94550 
Abstract

Introduction

Initial Physics Requirements for an MFTF

Thomson Scattering System . . . . . . . . . • . . . . . . 2

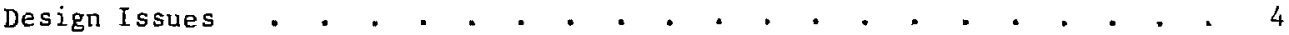

Thomson Scattering Power Spectrum . . . . . . . . . . . . 6

Background Power Spectrum , . . . . . . . . . . . . . 8

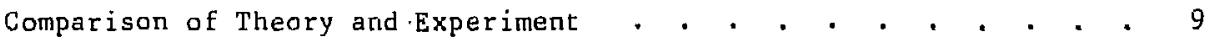

Scaling to MFTF . . . . . . . . . . . . . . . . . 12

Photon Statistics . . . . . . . . . . . . . . . . . 17

Laser . . . . . . . . . . . . . . . . . . 18

Glass-Laser Thomson Scattering . . . . • . . . . . . . . 21

Divergence in the Focal Zone . . . . . . . . . . . . . 23

Aligment in the Focal Zone . . . . . . . . . . . . . . 25

Objective: Lens or Mirror? . . . . . . . . . . . . . . 27

Radiation Environment . . . • . • . . . . . • . . . . 30

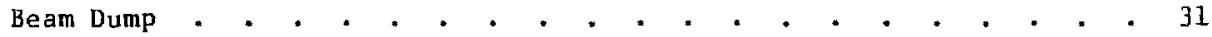

Polychromators . . . . . . . . . . . . . . . . . . 33

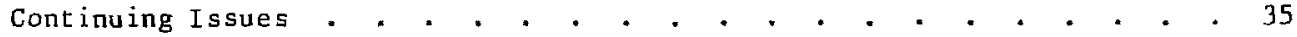

Laser . . . • . . . . . . . . . . . . . . . . 36

Background Radiation . . . . . . . . . . . . . . . . 36

Heat-loading of the Objective . . . . . . . . . . . . . . . . . 36

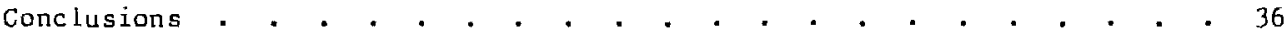

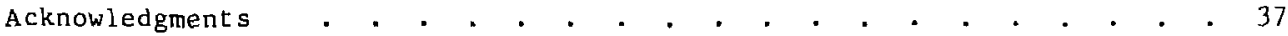

References . • • . • . . . . • . • • . . . . . . . . 38 


\section{ABSTRACT}

This report documents the design effort for a Thomson scattering diagnostic system for MFTF. The principal problem is obtaining enough photors, in the presence of a poorly known background, to make satisfactory measurements. No currently available laser will yield enough photons to do this. Design concepts for imaging and detection are discussed. The ability of components to survive in the high-radiation environment of MFTF is identified as an important problem. The transition to MFTF-b makes many of the problems identified here more serious.

\section{LNTRODUCTION}

The only nonperturbing technique for measuring electron temperature and density at a point in a plasma is Thomson scattering. In plasmas at temperatures greater than about $100-200 \mathrm{eV}$, x-ray spectra yield line-integrated temperature. Interferometry provides line-integrated densities.

Thomson scattering is the scattering of photons by electrons. For monochromatic photons, the spectrum of scattered radiation is a function of electron temperature $\mathrm{T}_{\mathrm{e}}$, and the intensity is a function of electron density $\mathrm{N}_{\mathrm{e}}$. With a laser as a photon source and with a limited region of observation, Thomson scattering gives point measurements of $T_{t}$ and $\mathbb{N}_{e}$. This is the primary cectinique for the measurement of $\mathrm{T}_{e}$ in fusion plasmas. ${ }^{1-3}$ since Thomson scattering is the only point measurement available, it is necessary for mapping temperature and density profiles.

Expected MFTF plasma parameters were used to establish a range of physical parameters and of locations at which measurements of $\mathrm{T}_{\mathrm{e}}$ and $\mathrm{N}_{\mathrm{e}}$ would be required. The computer code TOMSCAT ${ }^{4}$ was developed to analyze the measurement capabilities of Thomson scattering systems. This code was used extensively to compare the measurement capabilities of proposed system designs.

Both the physics requjrements and the design concept assume that a classical Thomson scattering design, such as that used on 2 XIIB and Tr $X$, would 
be applicable to MFTF. In fact, current Thomson scattering practice cannot meet MFTF requirements, but developments in laser and jetector technology and improvements in background-suppression techniques should make jt possible to meet MFTF requirements.

This report represents both the completion of work on MFTF and a transition point to MFTF-B. The plasma parameters on MFTF-B will be more difficult to measure, making the problems discussed in this report more significant.

INITIAL PHYSICS REQUTREMENTS FOR AN MFTF THOMSON SLATTERING SYSTEM

The initial physics requirements ${ }^{5}$ are that a Thomson scatcering measurement of electron temperature be made in plasmas witn densities of from $\mathrm{i} \times 10^{12}$ to $4 \times 10^{14} \mathrm{~cm}^{-3}$ and with electron temperatures up to $1 \mathrm{keV}$ (Fig. 1). Under ideal conditions the measurement accuracies must be at least

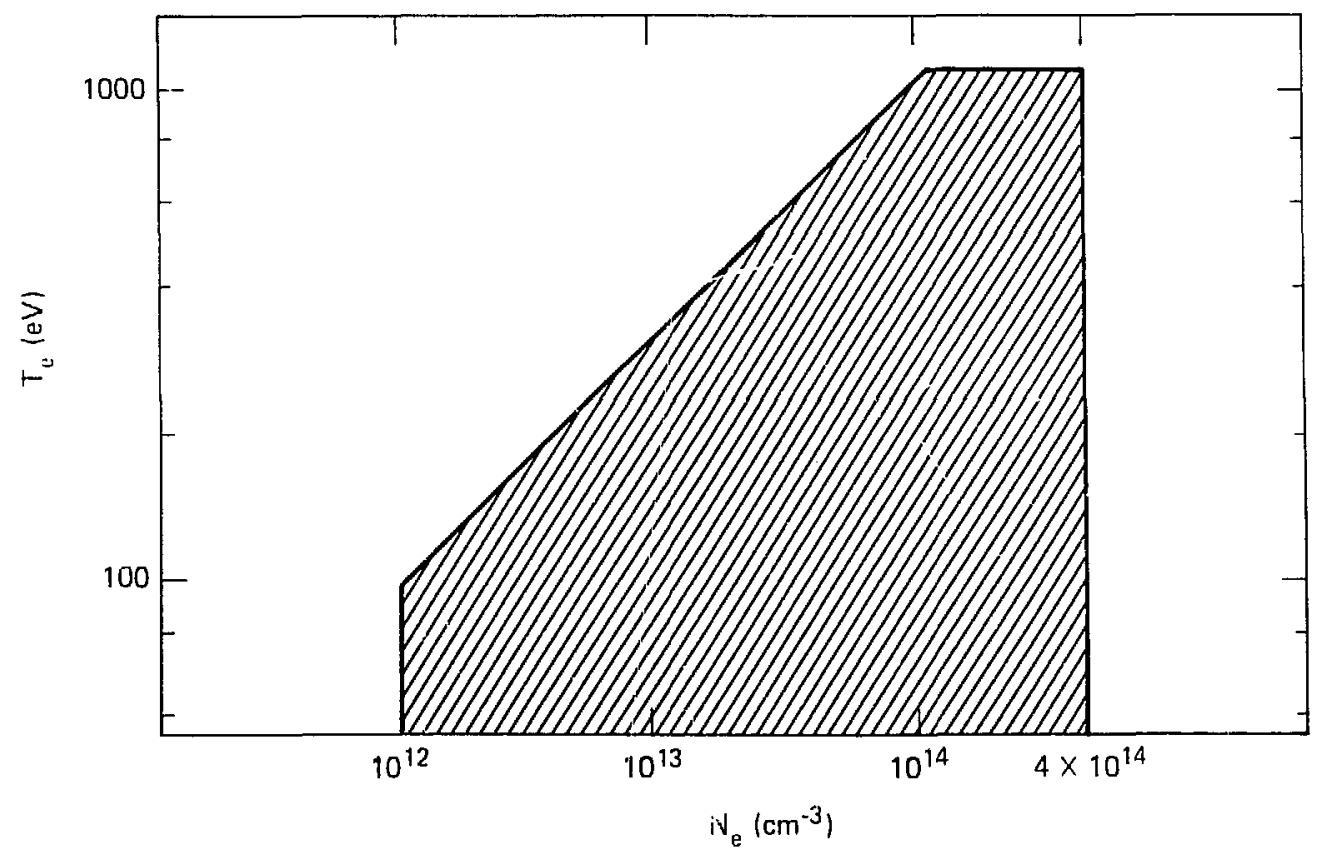

FIG. 1. The shaded area represents the parameter space within which Thomson scattering measurements are required on MFTF. 
$10 \%$ for $\mathrm{T}_{e}$ and $20 \%$ for $\mathrm{N}_{\mathrm{e}}$, with short-term repeatability of at least $10 \%$. The electron-density measurement requires calibration of the scattering system. Although the initial system is to look at only one point at any time, it must have the capability of simultaneously looking at as many as five points along the laser chord, as shown in Fig. 2 .

The initial requirement is to fire the laser once per machine shot, with a jitter of $\mathrm{l} \mathrm{ms}$ and at a time known to \pm 0.5 us. The data collection detectors and electronics must be capable of recording more than one laser pulse per machine shot. An upgrade requirement is that the laser be capable of multiple pulses during a machine shot. The system must function at a maximum rate of once every five minutes for as long as a full 8-hr shift. Routine alignment and laser aiming must be accomplished from outside the MFTF vault. It must be possible to sweep the laser beam to various points along

Meaurement points along laser chord through plasma
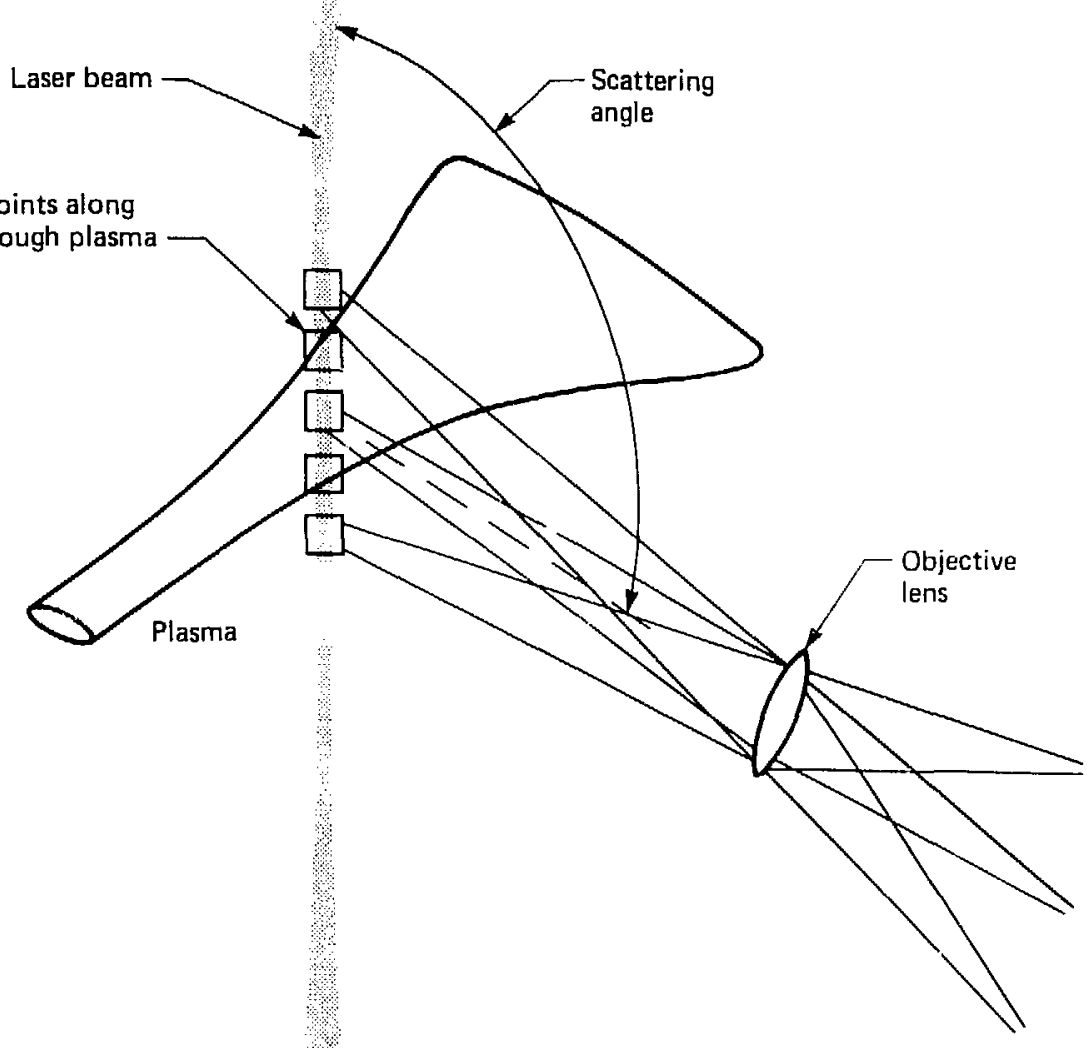

FIG. 2. Thomson scattering geometry. 
the $z$ axis, and to measure simultaneously as many as five points perpendicular to the $z$ axis. Figure 3 shows the required locations.

\section{DESIGN ISSUES}

The initial design approach for MFTF assumes that a classical 90-deg scattering system, similar to those used on 2XIIB and TMX, can meet the



FIG. 3. Required measurement locations, in machine-center coordinates. 
physics requirements. Other approaches, such as the use of forward scattering angles or drastically different wavelengths, are not considered here but must be considered for MFTF-B.

The typical system focuses a high-energy laser pulse along a line through the center of the machine (Fig. 4). The scattered radiation enters a lens system whose axis is perpendicular to the laser beam and is refocused onto an image plane just outside the diagnostic flange. Polychramators (spectrometer; detector combinations) in the image plane sample segments of the laser focal 1 ine.

MFCF is different enough from TMX in performance, environment, and magnitude that simple scaling of the TMX Thomson scattering diagnostic system makes litcle sense. We therefore reviewed the basic system parameters before proceeding with detailed design issues.

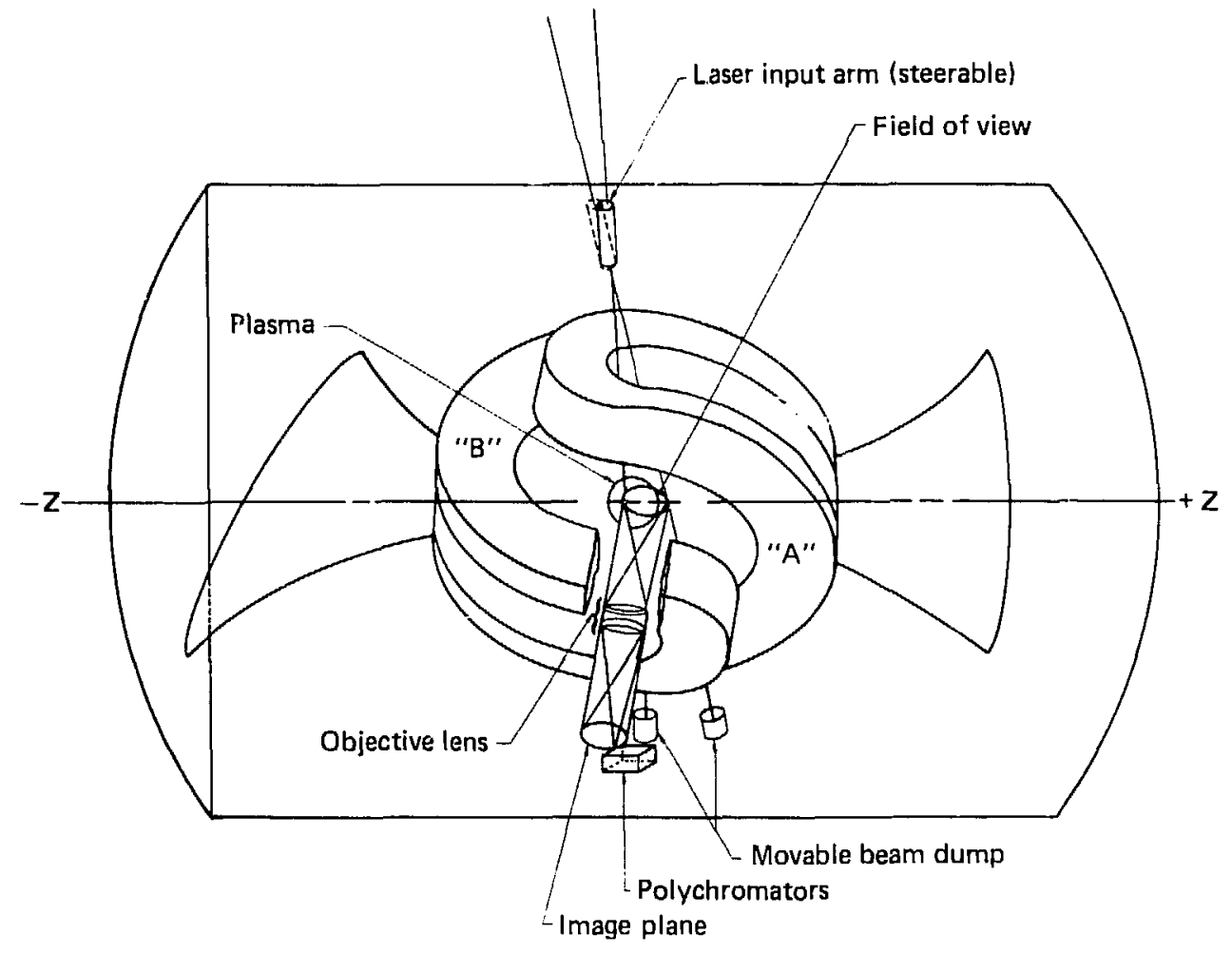

FIG. 4. First-try layout for a Thomson scattering diagnostic system in MFTF. (a) Looking north. 


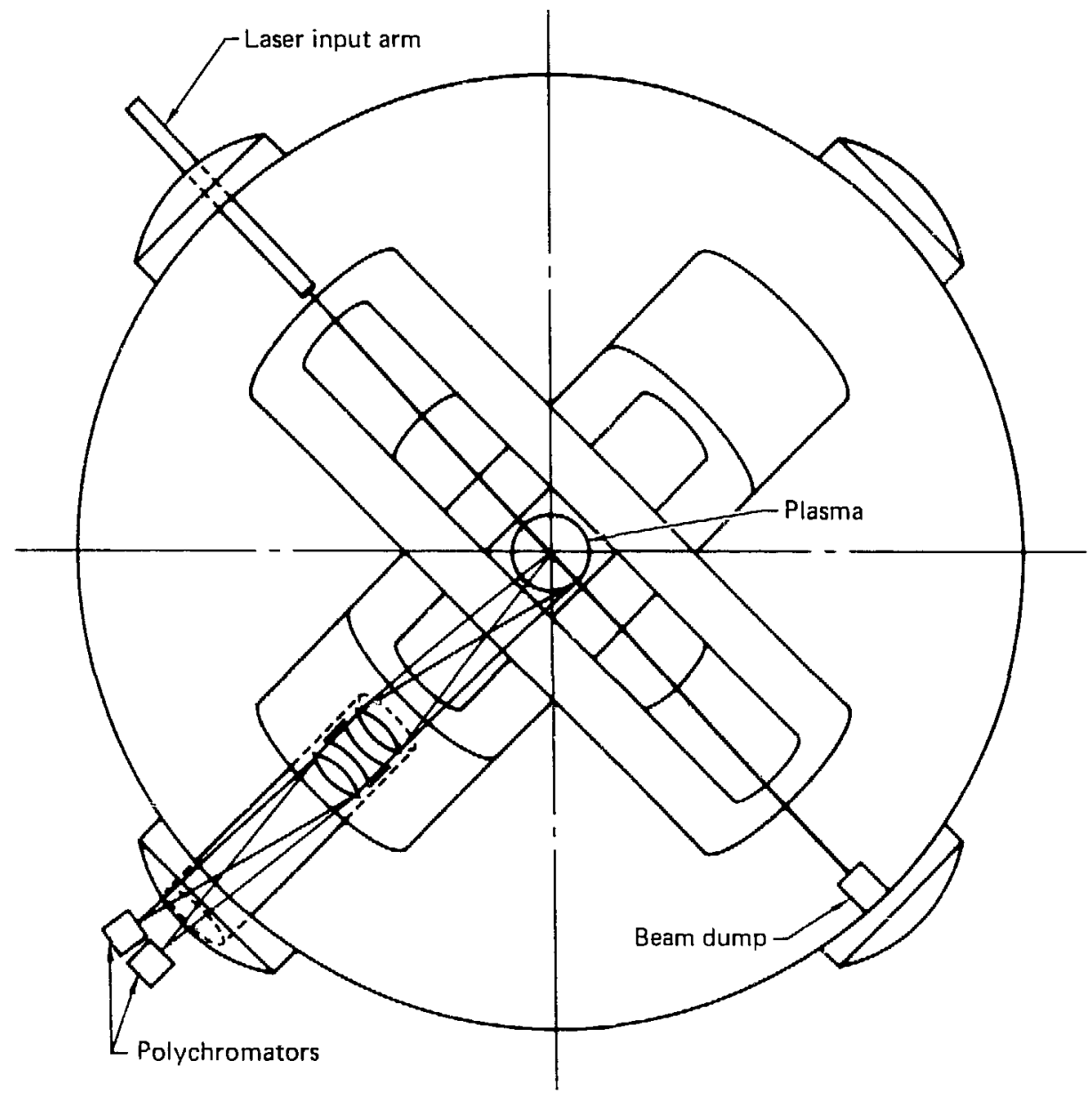

FIC, $4(b)$ Looking east.

THOMSON SCATTERING POWER SPECTRUM

The Thomson scatiering cross section $\sigma_{t}$ of the electron $1.3^{3}$

$\sigma_{t}=\frac{8}{3} \pi r_{e}^{2}=6.65 \times 10^{-25} \mathrm{~cm}^{2}$,

where $r_{e}$ is the classical electron radius, $2.82 \times 10^{-13} \mathrm{~cm}$. This cross section is so small that even at fusion densities of $10^{12}$ to $10^{14}$ 
electrons $/ \mathrm{cm}^{3}$ the fraction of light scattered is very small $\left(10^{-11}\right.$ to $10^{-13}$ per cm length of scatcering region); the Eraction scattered into a reasonable solid angle is smaller still. Thus high-power lasers and large-aperture collection optics are essential for Thomson scattering diagnostics.

To model the MFTF Thomson scattering power spectrum, we consider incoherent, relativistically-corrected scattering perpendicular to the polarization vector of the laser beau. The form of the power spectrum was derived by Goodman ${ }^{6}$ by carrying the angular dependence through the derivation of Mattioli and Papoular. 7 The angular dependence agrees, in the nonrelativistic limit, with the formula derived by sheffield. 8 The number of photons scatered into a solid angle $\Delta \Omega$ and unit wavelength $d \lambda$ is given by

$$
P_{S}(\lambda) d \lambda=\Delta \Omega \frac{d \lambda}{\lambda_{L}} r_{e}^{2} \frac{N_{e} e^{\Delta z}}{\sqrt{2} \pi \sin \theta / 2} \frac{P_{L} \lambda_{L}}{h c} \frac{c}{v} y_{l} \exp \left(-\frac{c^{2}}{v} \frac{y_{2}}{2 \sin ^{2} \theta / 2}\right),
$$

wh $: r=$

$$
\begin{aligned}
& A \text { = scattered wavelength, in } \mathrm{nm} \text {, } \\
& \Delta \sigma=\text { solid angle of observation, in } s r \text {, } \\
& \lambda_{\mathrm{L}}=\text { laser wavelength, in } \mathrm{nm} \text {, } \\
& r_{e}=\frac{e^{2}}{m_{e} c^{2}}=2.82 \times 10^{-13} \mathrm{~cm} \text { (classical electron radius), } \\
& N_{e}=\text { local electron density, in } \mathrm{cm}^{-3} \text {. } \\
& \Delta z=\text { lengti of observed scattering volume, in } \mathrm{cm} \text {, } \\
& \theta=\text { scattering angle, } \\
& P_{L}=\text { power of the laser beam, in } \mathrm{J} \text {, } \\
& \text { in = Planck's constant, } \\
& c=\text { speed of } 7 \text { ight, } \\
& v(\text { in } m / s)=\left(\frac{2 k T}{e}\right)_{e}^{1 / 2}\left(T_{e} \text { in } k\right)=5.93 \times 10^{5} T_{e}^{1 / 2}\left(T_{e} e^{i n ~ e V}\right) \text {, } \\
& r_{e}=\text { local electron temperature, }
\end{aligned}
$$




$$
\begin{aligned}
& y_{1}=\frac{1}{\sqrt{2}}\left[1-3.5 x+\left(7.63-\frac{0.25}{2 \sin ^{2} \theta / 2}\right) x^{2}-\left(13.07-\frac{0.63}{2 \sin ^{2} \theta ! 2}\right) x^{3}\right] \\
& y_{2}=\frac{1}{2} x^{2}(1-x), \\
& x=\frac{\lambda-\lambda_{L}}{\lambda_{L}} .
\end{aligned}
$$

BACKGROUND POWER SPECTRUM

To compure numbers of background photons due to bremsitrahlung, we derived the foll'wing expression from the work of Löchte-Holtgreven ${ }^{1}$ :

$$
\mathrm{P}_{B}(\lambda) \mathrm{d} \lambda=1.9 \times 10^{-37} \Delta \mathrm{V} \Delta \mathrm{t} \frac{\Delta \Omega}{4 \pi} \frac{\mathrm{d} \lambda}{\lambda} \frac{\mathrm{N}^{\mathrm{N}} \mathrm{i}_{\mathrm{g}} \mathrm{Z}_{\mathrm{eff}}^{2}}{h \mathrm{~h} \sqrt{\mathrm{T}} \mathrm{exp}}\left(\frac{-1239.5}{\lambda \mathrm{T}_{\mathrm{e}}}\right)
$$

where

$$
\begin{aligned}
& \Delta V=\text { collection tolume (see below) } \\
& \Delta t=\text { collection time } \\
& N_{i}=\text { ion density assumea equal to } \mathrm{N}_{e} \text { ) } \\
& \overline{\mathrm{g}}=\text { Gaunt factor } \\
& \mathrm{Z}_{\text {eff }}=\text { effective charge of ions (see below). }
\end{aligned}
$$

Other symbols in Eg. (2) were defined fior Eq. (1). The collection volume $\Delta V$ is the entire light-emitting region seen by the optics; we estimate $\Delta V$ by multiplying the area of the entrance slit, as imaged in the plasma, by the diameter of the piasma. For the Gaunt factor $\bar{g}$ we use a Born approximation, as given by Hughes ${ }^{9}$ :

$$
\vec{g}\left(\lambda T_{e}\right)=\frac{\sqrt{3}}{\pi} \ln \left(3.598 \times 10^{-19} \frac{\lambda T_{e}}{h c}\right) .
$$


It is important to note that the background radiation is collected from a chord across the entire plasma. Processes that dominate in the hot central region wili be different than those in the cooler outer regions, where recombination and impurity radiations are quite significant. The absolute intensity of the background is typically 5 to 100 times that expected fur deuterium bremsstrablung $\left(z_{\text {eff }}=1\right) \cdot 10$ This additional radiation is strongly dependent on machine cleanliness and varies substantially during a run cycle. Forrest et al., ${ }^{11}$ at Culham, used an enhancement factor of 100 $\left(z_{\text {eff }}=10\right)$ in their studies for JET.

\section{COMPARISON OF THEORY AND EXPERIMENT}

The computer code TOMSCAT was developed tc calculate both the Thomson scattering power spectrum and an enhanced-bremsstrahlung background power spectrum. To test the validity of TOMSCAT, we compared Thomson scattering data from a typical TMX shot with the code predictions. The TMX system has not been calibrated absolutely, so system transmission and detector quantum efficiencies are not known. The power spectrum at the collection lens aperture was therefore calculated, and the data were normalized to the curve. The electron temperature was determined by a best fit of the data, without relativistic correction. The density was measured with a microwave interferometer.

It is clear from the results, shown in Fig. 5, that the predicted scattering intensity closely resembles the data. The predicted background approximates the magnitude of tine actual background but does not show the observed structure. The stucture indicates thit the background is dominated by impurity line radiation. A spectral survey ${ }^{12}$ indicates the presence of strong $\mathrm{N}_{2}$ emissions at 647 and $679 \mathrm{~nm}$ (Fig. 6). Other $\mathrm{N}_{2}$ bants are probably present at lower intensities. The nitrogen is from vacuum or liner leaks too small to prevent machine operation. UV studies in TMX and other machines also indicate the presence of $\mathrm{He}, \mathrm{O}_{2}, \mathrm{~N}_{2}, \mathrm{C}$, and $\mathrm{F}$ as impurities that could emit in the ruby-laser Thomson scattering region of the spectrum, 620-720 nm. ${ }^{13,14}$ In MFTF, some leakage of helium from the magnets couid also be expected. 


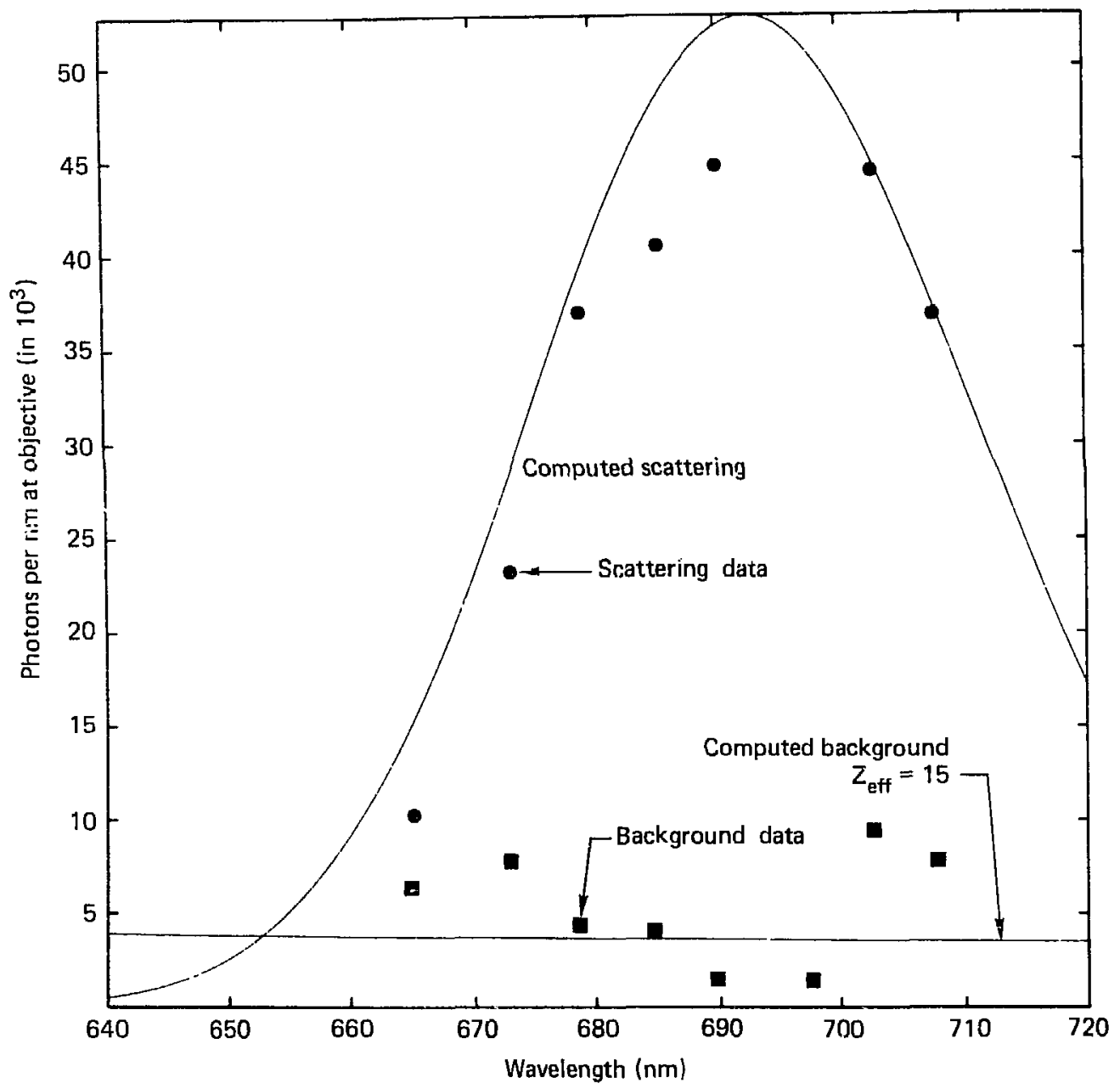

FIG. 5. Data points of a typical TMX shot compared with TOMSCAT computation. Solid circles are scattering data from shot 36, November 27, 1979; solid squares are background data. For the computed scattering curve, the following data were used:
$\mathrm{T}_{\mathrm{e}}=136 \mathrm{eV}$
$\mathrm{N}_{\mathrm{e}}=1.1 \times 10^{13} \mathrm{~cm}^{-3}$
$\mathrm{P}_{\mathrm{L}}=11 \mathrm{~J}$
$\theta=105 \mathrm{deg}$
$\begin{array}{rl}\tau_{p}=9 & \mathrm{~cm} \\ \tau & =50 \mathrm{~ns}\end{array}$ 


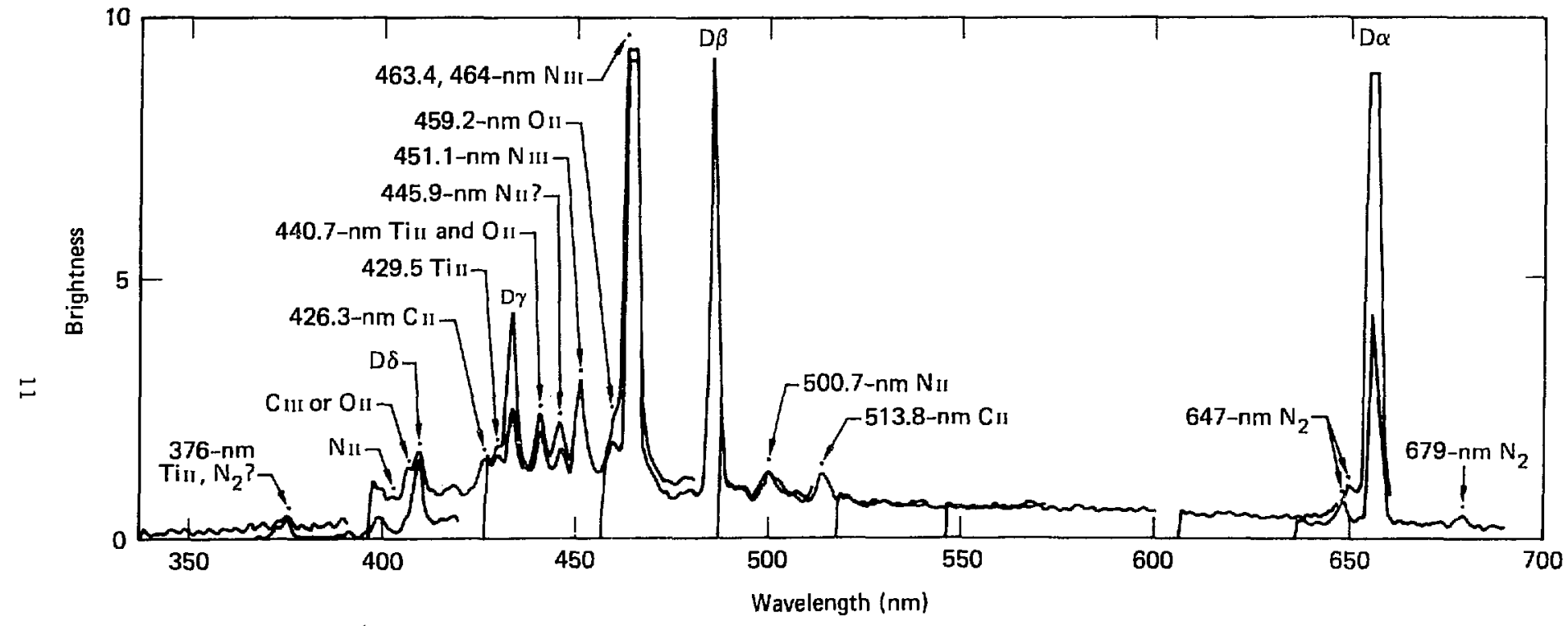

FIG. 6. TMX center cell survey spectrum, showing $\mathrm{N}_{2}^{+} 1$ ines at 647 and $679 \mathrm{tm}$. 
To see how the scattered-to-background ratio might scale to MFTF, we simplify Eqs. (1) and (2) to look at the important dependences, neglecting constants and exponential terms. For the scattered energy spectrum $P_{s}$ we have

$$
P_{s} \propto \Delta \Omega \frac{\Delta \lambda}{\lambda} \Delta z P_{L} \frac{N_{e}}{T_{e}^{1 / 2}},
$$

and For the background $P_{B}$ we have

$$
P_{B} \propto \Delta d \frac{\Delta \lambda}{\lambda} \quad \Delta V \Delta t z_{e f f}^{2} \frac{N_{e}^{N} i}{T_{e}^{1 / 2}} .
$$

Dividing the two equations, we get the ratio of scattered and background intensities,

$$
\frac{P_{S}}{P_{B}} \frac{\Delta z P_{L}}{\Delta V \Delta t z_{\text {eff }}^{2} N_{i}}
$$

Since $\Delta V=2 r_{p}$ $\Delta y \Delta z$, where $r_{p}=$ plasma radius and $\Delta y=$ entrance slit width, the signal-to-background ratio becomes

$$
\frac{P_{S}}{P_{B}} \propto \frac{P_{L}}{r_{P} \Delta y \Delta t Z_{e f f}^{2} N_{i}} \text {. }
$$

The geometric, temporal, and laset power terms in Eq. (3) are well understood, predictable, and independent of the model used to predict background level. The terms $\mathrm{P}_{L}, \Delta y$, and $\Delta t$ are system design parameters that can be adjusted tc improve the signa1-to-noise ratio. The terms $\mathrm{Z}_{\text {eff }}$ and $\mathbb{N}_{i}$ come directly from the enhanced-bremsstrahlung model and are not well understood for today's machines.

Evidence from TMX and 2XIIB indicates that true bremsstrahlung and recombination continua are not significant for plasmas of less than $20 \mathrm{~cm}$ diam with $\mathrm{N}_{\mathrm{e}}$ up to $10^{14} \mathrm{~cm}^{-3}$.

Although the assumption $Z_{\text {eff }}=10$ appears reasonable for $\mathrm{TMX}$ at a density of $10^{13} \mathrm{~cm}^{-3}$, it significantly overestimates the background for 
$2 X I I B$ at $10^{14} \mathrm{~cm}^{-j}$ ( see Fig. 7) and underestimates the 2 XIIB background for densicies of $10^{12} \mathrm{~cm}^{-3}$. In MFTF, with a plasma of $60-\mathrm{cm}$ diam and densities above $10^{14} \mathrm{~cm}^{-3}$, the bremsstrahlung background w111 become a limiting factor (Fig. 8).

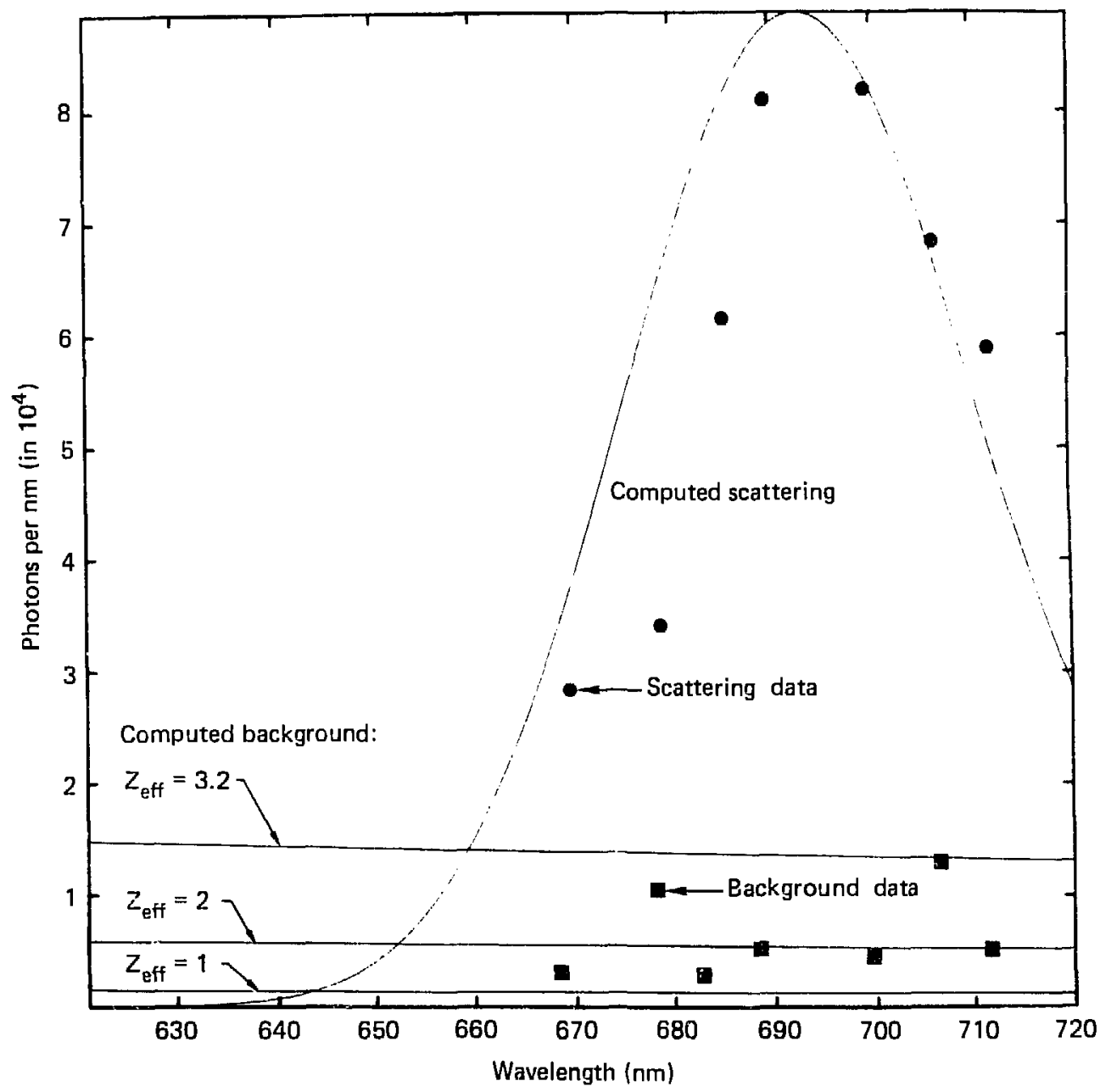

FIG. 7. TOMSCAT computation and data from a high-density $\left(\mathrm{N}_{\mathrm{e}}=10^{14} \mathrm{~cm}^{-3}\right)$ shot on 2XIIB. Soljd circles are scattering data from shot 26, September 12, 1977; solid squares are background data. For the computed scattering curve, the following data were used:
$\mathrm{T}_{\mathrm{e}}=134 \mathrm{eV}$
$P_{L}=3 \mathrm{~J}$
$\mathrm{N}_{\mathrm{e}}=1.0 \times 10^{14} \mathrm{~cm}^{-3}$
$r_{p}=6 \mathrm{~cm}$
$\theta=105 \mathrm{deg}$ 


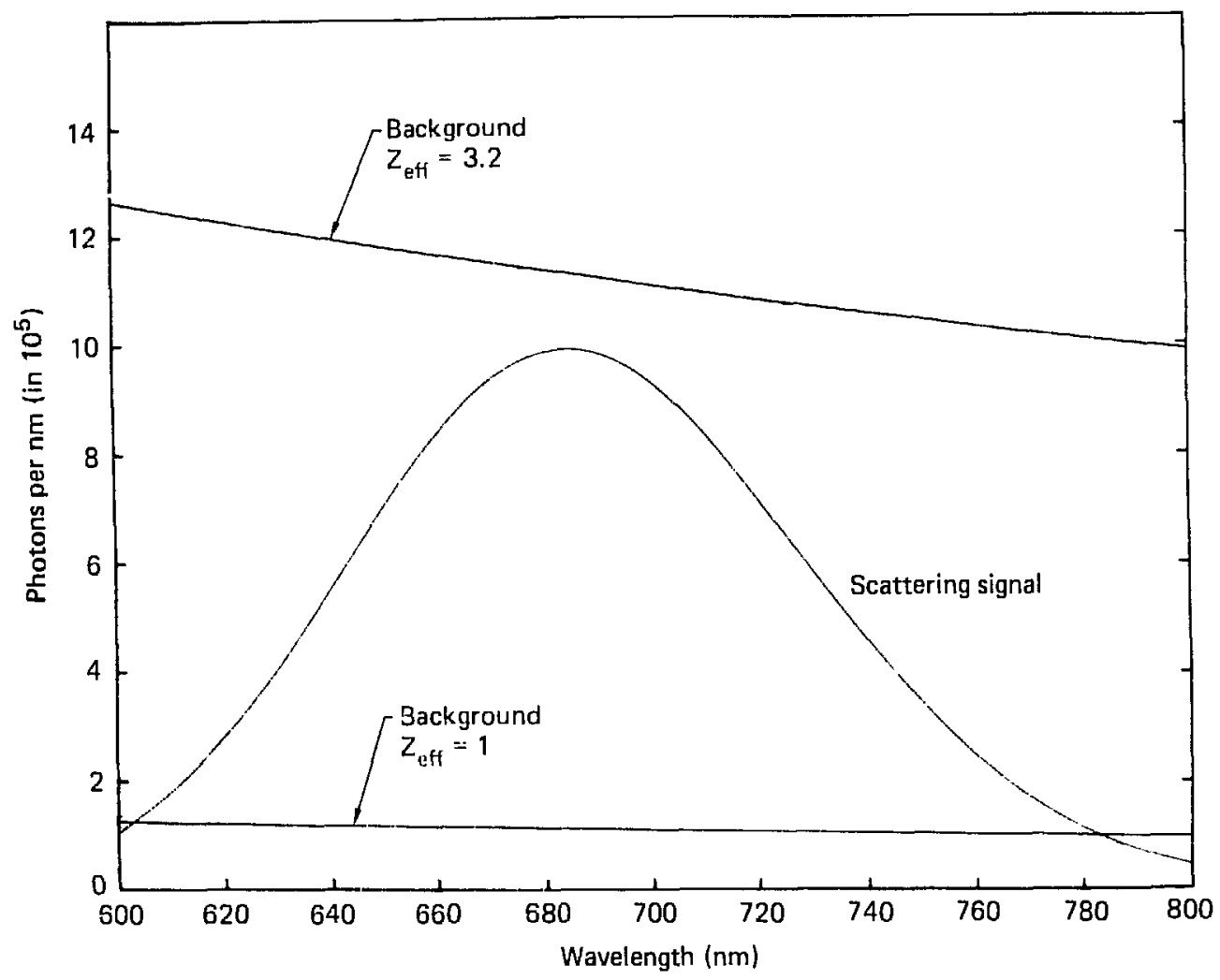

FIG. 8. TOMSCAT computation of MFTF Thomson scattering at high density. The fnllowing data were used:
$\mathrm{T}_{\mathrm{e}}=1 \mathrm{keV}$
$\mathrm{N}_{\mathrm{e}}=4.0 \times 10^{14} \mathrm{~cm}^{-3}$
$\mathrm{P}_{\mathrm{L}}=10 \mathrm{~J}$
$\theta=90 \mathrm{deg}$
$r_{p}=30 \mathrm{~cm}$

$\therefore$ wavelength of 694 nm and a $2 \times 20-\mathrm{mm}$ slit were assumed.

The background for electron densities below $10^{14} \mathrm{~cm}^{-3}$ appears to be spectral line radiation, mos': likely from species in low ionization states in the cooler parts of the machine. TMX and $2 X I I B$ experience does not tell us how the background scales with $\mathrm{T}_{\mathrm{e}}$ or $\mathrm{N}_{\mathrm{e}}$.

The enhanced-bremstrahlung model, Eq. (3), establishes lower limits of the background, particularly at higher densities. At lower densities, however, spectral emissions dominare, requiring a different model. If we assume that the impurity background is independent of $\mathrm{T}_{e}$ and $\mathrm{N}_{e}$, we can write a power spectrum of the form 


$$
P_{B} \propto \Delta \Omega \Delta V \Delta t \quad F(A),
$$

for $\mathrm{N}_{\mathrm{e}}<10^{14} \mathrm{~cm}^{-3}$, where $\mathrm{f}(\mathrm{A})$ is some complicated function of wavelength but is independent of $\mathrm{N}_{e}$ and $\mathrm{T}_{e}$. Dividing the scattered power spectrum by this background, we get the ratio

$$
\frac{\mathrm{P}_{\mathrm{S}}}{\mathrm{P}_{\mathrm{B}}}=\frac{\mathrm{P}_{\mathrm{L}} \Delta \lambda}{\mathrm{r}_{\mathrm{P}} \Delta y \Delta t \lambda \mathrm{F}(\lambda)} \frac{\mathrm{N}_{\mathrm{e}}}{\mathrm{T}_{\mathrm{e}}^{1 / 2}} .
$$

The dependence on plasma radius $r_{p}$ is questionable, since the source region is probably outside the hot central regions. However, it is probably as reasonable a scaling factor as any to model the source region.

To examine the measurement limits of a MFTF Thomson scattering system for comparison with the TMX system, we wish to plot lines of constant $P_{S} / P_{B}$ on a graph of $\mathrm{T}_{e}$ vs $\mathrm{N}_{\mathrm{e}}$. At lower densities, Eq. (4) indicates that the plot should follow lines of constant $\mathrm{N}_{\mathrm{e}} / \mathrm{T}_{\mathrm{e}}^{1 / 2}$.

Figure 9 shows the desired plot. To generate the figure, sample TMX data points were plotted, and a line of constant $\mathrm{N}_{\mathrm{e}} / \mathrm{T}_{\mathrm{e}}^{1 / 2}$ was plotted through the point with the lowest signal-to-noise ratio. The MFTF curves were scaled from the TMX curve using Eq. (4) and factors from Table 1. There is an upper density limit from the bremsstrálung, Eq. (3), which is independent of temperature to first order. The bremsstrahlung limits were estimated with TOMSCAT, requiring the peak of the scattering spectrum to fall below the

\begin{tabular}{|c|c|c|c|}
\hline & TMX & \multicolumn{2}{|c|}{ MFTF } \\
\hline & Ruby laser & Ruby laser & $\begin{array}{c}\text { Doubled glass } \\
\text { laser }\end{array}$ \\
\hline$\lambda_{L}(n m)$ & 694 & 694 & 532 \\
\hline$P_{L}(J)$ & 10 & 10,25 & 25,65 \\
\hline$r_{p}(\mathrm{~cm})$ & 9 & 30 & 30 \\
\hline$y(\mathrm{~mm})$ & 1 & 2 & 2 \\
\hline$t$ (ns) & 50 & 50 & 25 \\
\hline $\mathrm{E}_{\text {no }}(\Delta \Omega)$ & 5 & 5 & 5 \\
\hline
\end{tabular}

TABLE 1. TMY/MFTF scaling factors. 


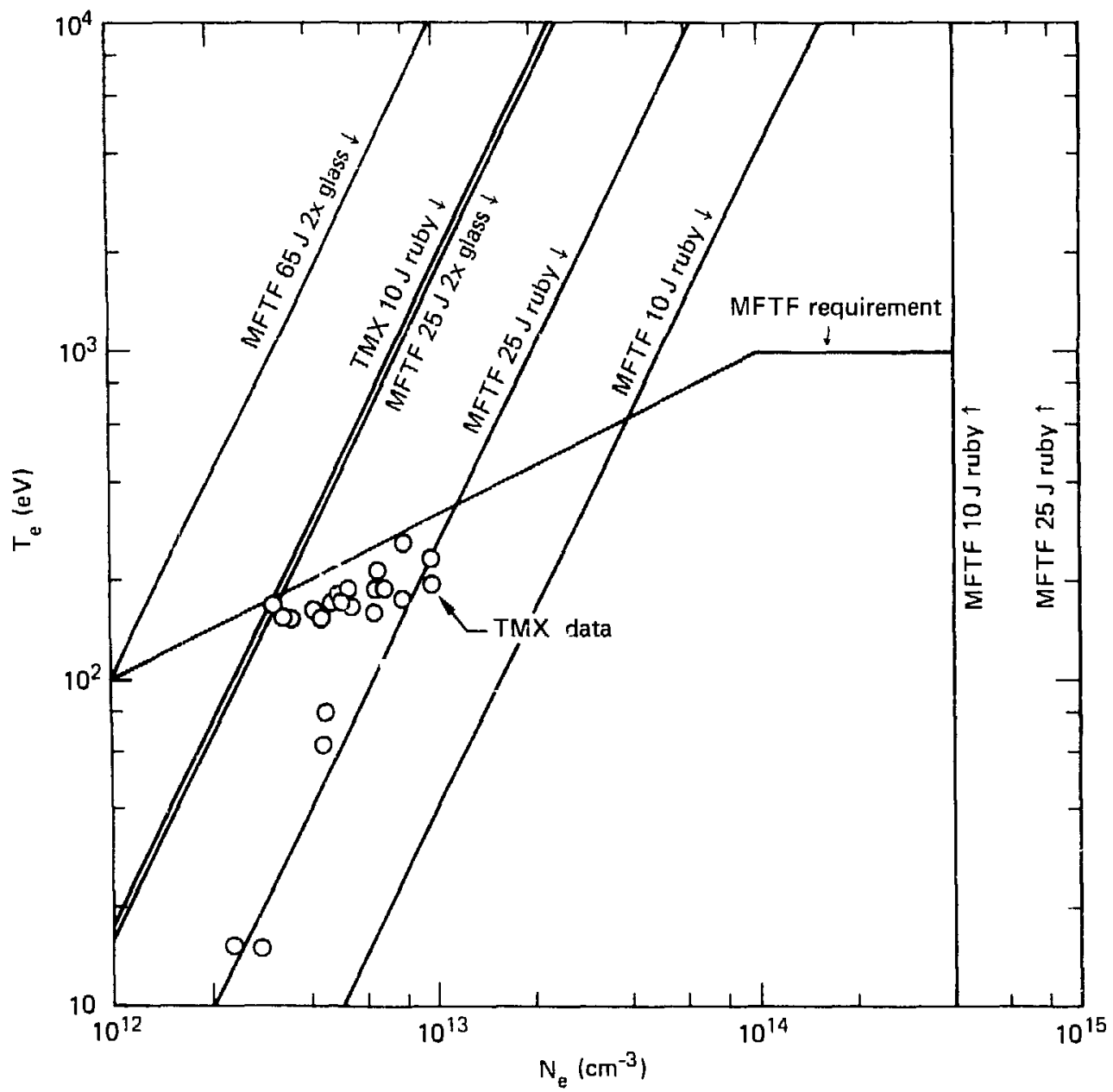

FIG. 9. Comparison of measurement-capability limits of proposed Thomson scattering systems with MFTF requirements. Circles are TMX data points. An $\mathrm{f} / 5$ system was assumed. Diagonal $\mathrm{l}$ ines are $\mathrm{L}$ ines of constant $\mathrm{T}_{e} / \mathrm{N}_{\mathrm{e}}^{1 / 2}$; vertical lines are bremstrahlung-dominated density limits. See text for discussion. The artow associated with each plotced line indicates the region in which satisfactory measurements can be made.

ten-times-enhanced bremsstrahlung curve $\left(z_{\text {eff }}=3.2\right)$. Clearly much of the MFTF nperating region will not be measurable with present 10-J ruby laser technology, and part of the range will be inaccessible even with $25-\mathrm{J}$ frequency-doubled glass lasers. 
The number of photons collected for both the signal and background determines the actual measurement limits. The collection soljd angle and the collection and detection efficiencies were taken as constants in the scaling of the previous section. These factors drop out of both Eqs. (3) ano (4). However, at low density, the ability to subtract the background from the signal is limited by photon statistics. That is, the uncertainty of the detected light level is $n^{l / 2}$, where $n$ is the number of detected photons. This implies that at least 100 scattered photons must be detected to achieve an uncertainty of $10 \%$. In the presence of $n_{b}$ background photons, the uncertainty $\Delta n_{5}$ in the number of scattered photons is

$$
\Delta n_{s}=\left(n_{s}+n_{b}\right)^{1 / 2}=n^{1 / 2} .
$$

This means that the uncertainty of the scattered signal is the same as the uncertainty of the total detected signal. The total number of phocons detected can be expressed as

$$
\mathrm{n}=\left(\mathrm{P}_{\mathrm{S}}+\mathrm{P}_{\mathrm{B}}\right) \Delta \Omega \mathrm{Tq} \Delta \lambda
$$

where

$$
\begin{aligned}
& \Delta \Omega=\text { solid angle of collection, } \\
& T=\text { optical system transmission, } \\
& q=\text { detector quantum efficiency, }
\end{aligned}
$$

We have

$$
\Delta \Omega=2 \pi(1-\cos \theta)=2 \pi\left(\frac{\theta^{2}}{2 !}-\frac{\theta^{4}}{4 !}+. . \cdot\right),
$$


but

$$
\theta=\tan ^{-1} \frac{1}{F_{n o}}=\frac{1}{E_{n o}} ;
$$

thus

$$
\Delta \Omega=\frac{\pi}{\mathrm{f}_{\text {no }}^{2}},
$$

where

$\mathrm{F}_{\text {no }}=$ focal ratio of objective lens.

Thus, finally,

$$
n=\left(P_{S}+P_{B}\right) \frac{\pi T q \Delta \lambda}{f_{\text {no }}^{2}} .
$$

Estimated performance for TMX-U using the value $\mathrm{T}_{q}=0.03$ is given in Fig. 10. With channels 5 to $10 \mathrm{~nm}$ wide, it is questionable whether enough photons can be collected to overcome the background. A fourfold improvement of the detector quantum efficiency $q$ is expected for TMX upgrade with the installation of GaAs photocathode PMT's. This should substantially improve the accuracy of measurements at electron densities near $10^{12} \mathrm{~cm}^{-3}$.

\section{LASER}

Thomson scattering measurements on magnetically confined fusion plasmas have generally been performed using ruby lasers with 20-ns pulses of up to $10 \mathrm{~J}$. These lasers are clearly inadequate for MFTF, as Fig. 9 shows; larger lasers must be considered.

The performanse requirements for the laser come primarily from Eqs. (3) and (4). With background conditions simjlar to those in TMX, MFTF will require an intensity of about $3 \times 10^{9} \mathrm{~W} / \mathrm{mm}^{2}$ delivered into the measurement volume. Further requirements are that the measurement volume be $60 \mathrm{~cm}$ long 




FIG. 10. Computed response of TMX Thomson scattering system at iow density.

and that the laser be pulsed every 5 min for 8-hr shifts. No existing lasers meet all these requirements.

Candidate lasers fall into two categories: those that are close to MFTF requirements and those with the potential of meeting those requirements. Those in the "close" category include neodymium-glass lasers ( $\lambda_{0}=1064 \mathrm{~nm}$ ), frequency-doubled neodymium-glass lasers $\left(\lambda_{0}=532 \mathrm{~nm}\right)$, and ruby lasers $\left(\lambda_{0}=694.3 \mathrm{~nm}\right)$. Those in the "potential" category include lasers using alexandrite $\left(\lambda_{0}=755 \mathrm{~nm}\right)$ and the rare-gas halides ( $\wedge_{0}$ typically near-UV). 
The solid state lasers are damage-limited. That is, there is a maximum energy density above which laser damage will occur (cypically $5 \mathrm{~J} / \mathrm{cm}^{2}$ for ruby at $20 \mathrm{~ns}$ (pulse). Thus the laser energy is limitea by the cross sectional area of the available laser material. As the laser rnds or discs become larger, the cooling time between shots increases. While the laser is cooling its optical quality is degraded, giving the beam a higher divergence. The increase of divergence at higher repetition rates becomes a serious problem for larger lasers.

The gemstone alexandrite is a chromium-doped chrysoberyl (BeA $1_{2} 0_{4}$ ) discovered in Russia on Caar Alexander's birthday in 1843. Laboratory-grown alexandrite is being developed as a Laser medium by Allied Chemical Corp. Alexandrite can operate as a thrte-level iaser at $680 \mathrm{~nm}$ or as a four-level tunable laser at about $755 \mathrm{~nm}$. Its gain is about four times higher than ruby, and it has better thermal properties. Allied Chemical is going onto the market with 1-cm-diam rods, with a maxinum output power as $5 \mathrm{~J}$, and is waiting to assess the inarket before expanding its crystal growing tacilities.

Rare-gas halide excimer (RGH) lasers have been under development for several years, and several companies now have such lasers on the market. Experimental work at LLNL, AVCO, and elsewhere is directed at scaling to high powers. These are discrarge-pumped or E-beam-pumped gas lasers, so the limiting factors tend to be in the pumping technology. These lasers have severe problems with beam quality and pulse length, but the problems shoula be surmountable with a Raman compression cell developed at LLNL. Probably a more severe limication of the RGH lasers is that their output is in the UV. typically at 249,308 , or $350 \mathrm{~nm}$. This region is rich in spectral line emission from the plasma, and the bremstrahlung intensity in this region is at least double that at ruby wavelengths. It is difficult to fabricate damage-resistant, low-scattering optical components for these wavelengths.

Thus, although there is significant effort in RGH laser technology, such lasers are not likely to become useful as Thomson scatcering sources in the near future.

Ruby iasers, commonly used in Thomson scattering measurements on fusic: plasmas, are linited to 10-J pulses by their 19-mm maximum diameter. The ruby rod manufacturer, Union Carbide, has just expanded its ruby growing capability to provide 25-mm core-free rods. Quantel Laser Corp., under contract from Oak Ridge National Laboratory, is trying to build a 25-J laser with the new $25-\mathrm{mm}$ 


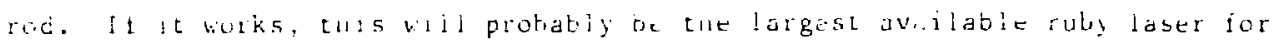
a tew yua:s.

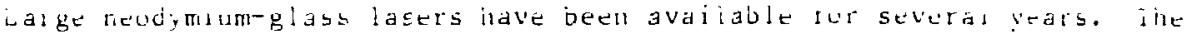
recent avalubility oI large-apercure frequency-aubling crystals nas aroused consjetable incerest in glass as a Thomson scatcering sourct Quantel has uelivered several lasels capable of B0-J, 3-to-6-ns pulses at i064 nm. Frequelicy ruubled, these produce $25 \mathrm{~J}$ at $5 \mathrm{~s}$ ? $\mathrm{nm}$. Experimental lasers at LLNL ilave delivereo l-ns pulses of up to $100 \mathrm{~J}$ at $532 \mathrm{~mm}$.

The new phosphate-glass laser iods carrently replacing the sllucats iaser glasses have betcer thermal and opticai properties, so repetstall rate ma: nut be at sirious probiem. The Quantei system operates at one shot per minute usillg +5-mm-diam rods. Quantel expects that its $75-m m$ rods bili recycle in i mili and deliver about $250 \mathrm{~J}$ at $1064 \mathrm{~nm}$ or $80 \mathrm{~J}$ at $53 \mathrm{~nm}$.

The lanoratory for Laser Energetics at the University of Rochoste uses rus ol ip to !00-mm diam, hut the divergence. repetition-rate reiationsiap for tnelr system is not clear. The 100-mm disc amplifiers usez at LLNL, hohever, ilave very long cooling times--about $30 \mathrm{mir}$.

Tire great acvantage of glass lasers is the continuing technological development in tirat area; ruby-laser technology has changed lattie in the iast tive yars. A Thomson scatering system using the maximumenergy ruby laser $(25 \mathrm{~J})$ woula not meet MFTF requirements, while a frequency-doubled glass system with the same pulst energy would come close because ot its shorter pulse length (Fig. 9). This results from the collection-time deperrence in Eqs. (3) and (4). Furthermore, a glass system coulube expandable for th. highr: t expected on MFTF-B (Fig. 11,

\section{GLASS-LASER THOMSUN SCATTERING}

Several groups are working on glass-laser Thomson scattering. The first successful results were published by Peterson et al. ${ }^{5}$ of Pennsylvania State University. The Peterson system uses a frequency-doubled glass laser, operating at $532 \mathrm{~nm}$, with $\mathrm{S}-20$ photomultiplier tubes as detectors, to measure a 10-cm-diam theta-pinch plasma with $\mathrm{N}_{\mathrm{e}}=10^{16} \mathrm{~cm}^{-3}$ and $\mathrm{T}_{\mathrm{e}}=10 \mathrm{eV}$.

Hursch et al., 16 ir Stuttgarl, are investigating avalanche photodiodes for use directly at $1064 \mathrm{~nm}$. These detectors have high quantum efficiencies about $75 \%$, but they also have high noise levels. They can be used only by integrating over long wavelength intervals to provide enough signal to 
(a)

$$
\underbrace{\text { Osc P.C. Etalon S.A. }}_{6 \times 170 \mathrm{~mm}} \underset{6 \times 120 \mathrm{~mm}}{=}
$$

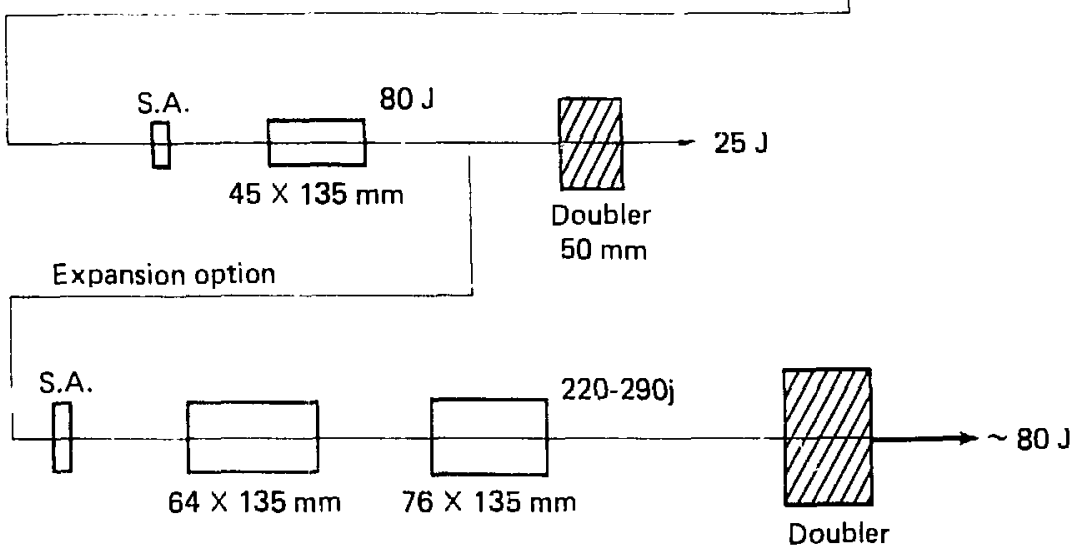

$75 \mathrm{~mm}$

(b)

Eg Gen photonics

Expansion option S.A.

P.C. Pockels cell

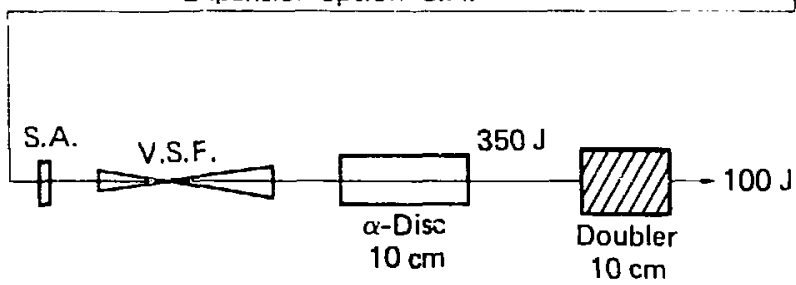

S.A. Saturable absorber

V.S.F. Vacuum spatial filter

FIG. 11. Possible frequency-doubled glass laser systems for use on MFTF.

(a) Quante! design. The $25-\mathrm{J}$ output version would cost about $\$ 150,000$; the $80-J$ expanded version would cost about $\$ 250,000$. (b) ILC/LLNL design. The 25-30 J output version would cost about $\$ 400,000$; the $100-\mathrm{J}$ expanded version would cost about $\$ 600,000$. 
overcome the noise. Thus they appear to be most useful in two-channe! systems, such as that preposed by Lasalle ard Platz. 17

Forrest et al., 11 at Culham, propose that a two-wavelength system be used for JET. Frequency-áoubled glass lasers at $532 \mathrm{~nm}$ would be used for low $\mathrm{T}_{\mathrm{e}}$ an: ordinary glass lasers at $1064 \mathrm{~nm}$ for high $\mathrm{T}_{\mathrm{e}}$. It is worth noting that at high electron temperatures $\left(\mathrm{T}_{\mathrm{e}}>2 \mathrm{keV}\right)$, a significant number of photons are scattered from $1064 \mathrm{~nm}$ into the region above $800 \mathrm{~nm}$, in which photomultiplier tubes are useful.

The Stuttgart group also Droposes to look at extreme forward scattering angles with a nigh-repetition-rate $(100 \mathrm{~Hz})$, low-resolution system.

At forward scattering angles $\theta$, the factor $1 /(\sin \theta / 2)$ in $\mathrm{Eq}$. (1) significantly increases the scattering into a given solid angle. It will probably be necessary to go to forward-angle scattering for MFTF-B, even though this will lead to other problems.

DIVERGENCE IN THE FOCAL ZONE

Both bremstrahlung- anii spectral-1ine-dominated signal-to-background ratios (Eqs. (3) and (4), respectively) are inversely proportional to the width of the collection region. That is, we have in either case

$$
\frac{\mathrm{P}_{\mathrm{S}}}{\mathrm{P}_{\mathrm{B}}} \propto \frac{1}{\Delta \mathrm{y}} .
$$

Reduction of the size of the collection region directly inceases the signal-to-noise ratio as long as the signal is not reduced. The ideal collection region is just the scattering region--the region occupied by the laser beam.

The width of the collection region is definea by tine width of the entrance slit of the spectrometer on which the laser focal zone is imaged. We cannot make the collection region as small as we please, because there is a minimum diameter to which we can focus the laser beam, and becausa the image of the collection region cannot be placed on the entrance slit with perfect accuracy and stability.

Both the divergence of the laser and the geometric focal ratio contribute to the width of the laser focal zone. The divergence of the laser depends on 
the beam diameter, which is usually limited by the mode structure of the laser but is ultimately limited by diffraction. The geometric focal ratio becomes significant away from the waist of the laser beam, but it also determines the laser beam diameter at the last focusing lens and thereby affects the divergence. We define the focal ratio $\mathrm{f}_{\text {no }}$ as the focal length of the focusing lens, $F$, divided by the l/e diameter of the beam at the lens, $d_{w}$ : that is,

$$
f_{\text {no }}=\frac{E}{a_{w}}
$$

The beam diameter $d(z)$ at distance $z$ from the beam waist can then be written as

$$
\mathrm{d}(\mathrm{z})=20 \mathrm{f}_{\mathrm{no}} \lambda+\frac{\mathrm{z}}{\mathrm{f}_{\mathrm{no}}}
$$

where $\delta$ is the divergence expressed as a multiple of the diffraction limit.

MFTF requires that the useful focal region be $40 \mathrm{~cm}$ long, so as to span radial positions from -10 to $+30 \mathrm{~cm}$; this gives $z=20 \mathrm{~cm}$. A maximum beam width of $2 \mathrm{~mm}$ was initially chosen as a useful working tolerance based on Eqs. (3) and (4) at reasonable laser powers. For ruby lasers, $\lambda=694 \mathrm{~nm}$. With these values inserted, Eq. (5) was solved quadratically for $f_{\text {no }}$ for various laser divergences. The results are given in Table 2. For divergences greater than 3.6 , the solutions become complex.

The multimode lasers used on 2 XIIB and TMX are about 25 times diffraction limited (that is, $\delta \simeq 25$ ) and therefore cannot meet the MFI'F focal-zone requirements. Bretz ${ }^{18}$ has put considerable effort into achieving single-mode, low-divergence output from ruby lasers. The state of the art for ruby lasers is about three times diffraction limited. Frequency-doubled giass lasers have an advantage over ruby because of their $30 \%$ shorter wavelength, and single-mode lasers are typically about 3 times diffraction limited. Undoubled glass lasers, although nearly diffraction-limited, have nearly a factor of 2 wavelength disadvantage. The precise divergence for any laser varies with operaring conditions, repetition rate, etc.

It should be pointed out that if one uses the smaller value of the focal ratio of the two given for each value of $o$ in Table 2 , the beam diameter at the waist can be made substantially smaller than at $20 \mathrm{~cm}$. This would allow one channel to be substantially more sensitive. The data in Fig. 9 were 
TABLE 2. Solutions of Eq. 5 requiring a beam diameter of $2 \mathrm{~mm}$ at $20 \mathrm{~cm}$ from the beam waist, with wavelength $\Lambda=694 \mathrm{~nm}$, for various laser divergences $\delta$.

\begin{tabular}{lll}
\hline 0 & $\begin{array}{l}\mathrm{d}(0) \\
(\mathrm{mm})\end{array}$ & $\mathrm{f}_{\text {no }}$ \\
\hline 1 & 0.14 & 108 \\
1.9 & 1341 \\
2 & 0.33 & 120 \\
& 1.67 & 604 \\
& 0.58 & 141 \\
& 1.42 & 343 \\
\hline
\end{tabular}

computed for the 2-mm slit width; thus even with a three-tines-diffraction laser the center channel could he pushed to a $T_{\text {e }}$ limit about an order of magnitude higher than those shown.

\section{ALIGNMENT IN THE FOCAL ZONE}

The image of the laser-beam focal zone must fall precisely on the entrance slit of the spectrometer or some of the signal will be lost. On IMX, the entire laser and optical system was carefully mounted on a rigid structure; this approach to mounting is impossible on MFTF, so an active alignment system will be required. On TMX, the entrance slit was made wider than the laser-beam image to allow for alignment errors and drift; because of the background problem in MFTF, a small slit width will be essential.

On TMX an alignment flag, mounted and referenced to the magnet, can be flipped in to define machine center. The laser and viewing systems are manually aligned to the flag about once a day. There is no provision for measuring the actual focal point or beam diameter at machine center.

A multidetector array placed across the image of the laser beam in the focal plane of the entrance slit would give both position and beam width information on every shor (Fig. 12). Since the detector is looking at scattered radiation, it would only give a signal when a plasma was present, 
(a)



(b)

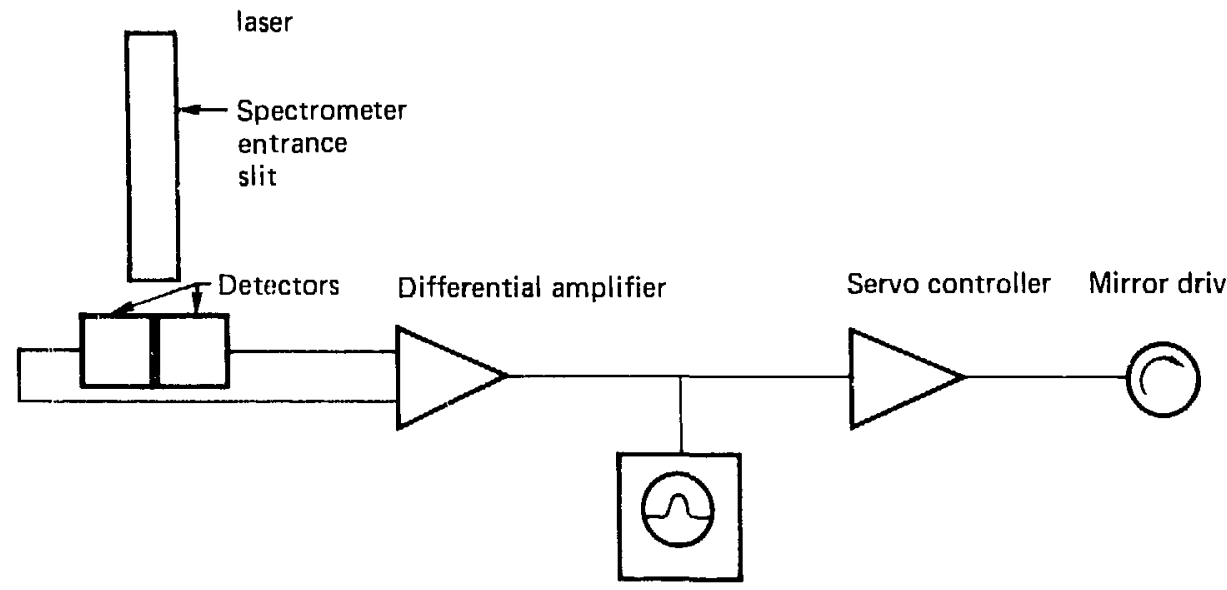

FIG. 12. Autoalignment and focus systems. (a) Linear detector array, capable of recording beam position and beam width. (b) Two-element array, capable of recording only beam position.

when there was a poor vacuum (about 1 Torr, or $100 \mathrm{~Pa}$ ), or when a target was inserted. During normal machine operation the detector signal could be used with a steering mirror to co:rect the laser's position for the next shot. The beam-width measurement would allow optimization of both laser focus and entrance-slit width. 
OBJECTIVE: LENS OR MIRROR?

The objective is the optical element that collects the scattered light and focuses it onto the entrance slit of the spectral analyzer. Two physical constraints determine the nature of the objective: these are the required collection solid angle and the required field of view. In addition to the physical requirements, the mechanical constraints and operating conditions of the machine impose severe limitations on the design.

The collection solid angle $\Delta \Omega$ is given by

$$
\Delta u=\frac{\pi}{f_{0}^{2}},
$$

where $E_{0}=R_{0} / A$ is the focal ratio of the objective; here $A$ is the aperture of the lens and $R_{0}$ is the distance of the lens from the object (the laser focus).

The TMX objective has a focal ratio of 4.9 and a magnification of 1.25 . It would be difficult to do better on MFTF, so a focal ratio of 5 was set as a design goal.

The field of view (FOV) is defined here as the diameter of the minimum circle, in a plane perpendicular to the objective in the plane cif the laser Leam, that incorporates the required measurement points. That is, the FOV is the minimum circle that can be drawn around the points of Fig. 3 .

The physics requirements specify a $50 \times 55-c m$ array of points, thus requiring a $75-\mathrm{cm}$ FOV. Off-axis aberrations and vignetring (obscuration) 1 imit the FOV.

The magnification of the objective is limited, both by our inability to make large optics and by the lack of space in and around the machine. Magnification is defined as the ratio of the image and object sizes $i$ and $o$ :

$$
m=\frac{i}{o} \text {. }
$$

The magnification also determines the ratio of the back and front focal lengths.

For the MFTF objective, the magnification is limited roughly to the range 0.5 to 2. The lower 1 imit comes from the requirement of a front focal ratio $F_{0}$ of 5 . The optics become more complex and expensive as the focal ratio 
gets lower; $f / 2$ or $f / 2.5$ is the practical lowet 1 imit for spectral systems. The upper 1 imit comes from size of the image plane and the clear region necessary to project the image out of the machine.

At close conjugates $(m \simeq 1)$, mirror systems require apertures at least 20 times larger than the Fov because of uncorrectable aberrations. A mirror system with a FOV of $75 \mathrm{~cm}$ would therefore require an aperture nearly three times that of the Mt. Palomar 200-in. telescope! Such a system would not fit within either the MFTF vault or the MFTF budget. Thus it is not reasonable to use mirrors to simultaneously cover the entire required field of view.

A mirror system (Fig. 13) with an aperture of $0.75 \mathrm{~cm}$ would have a useful FoV of less than $5 \mathrm{~cm}$. This might be acceptable for a single-point measurement if the system could be moved on a shot-to-shot basis to survey the required FoV. At $m=1$, a Cassegrain system would be $70 \%$ obscured by the

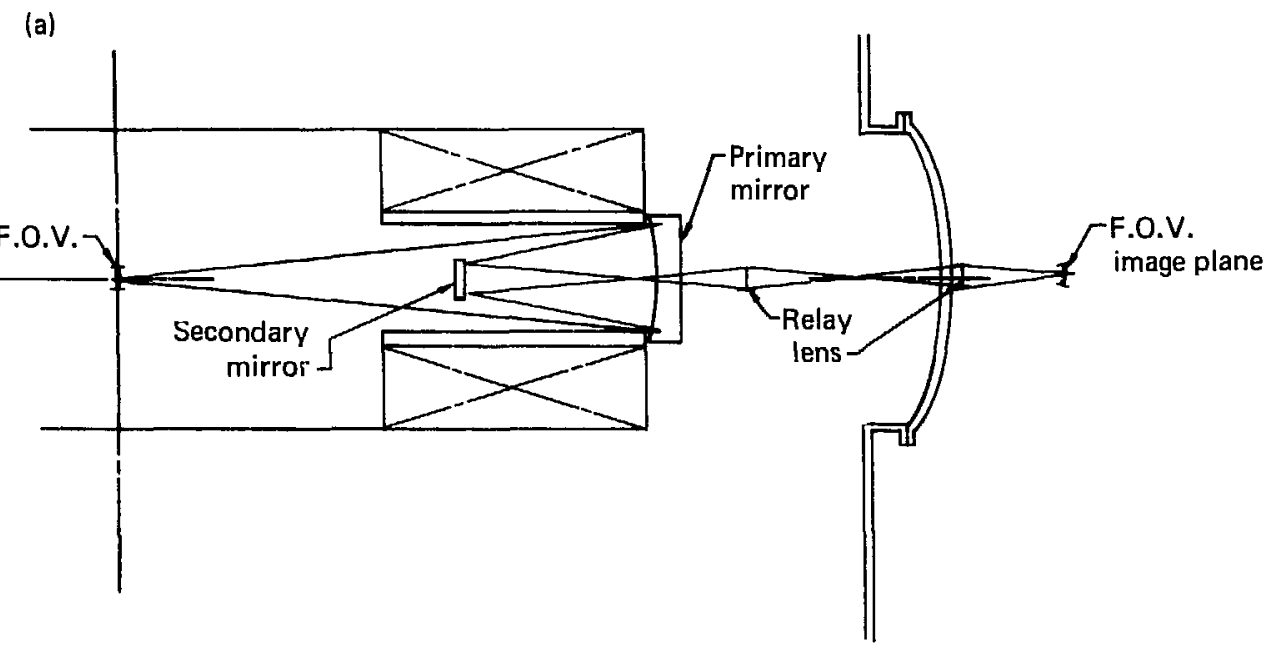

(b)

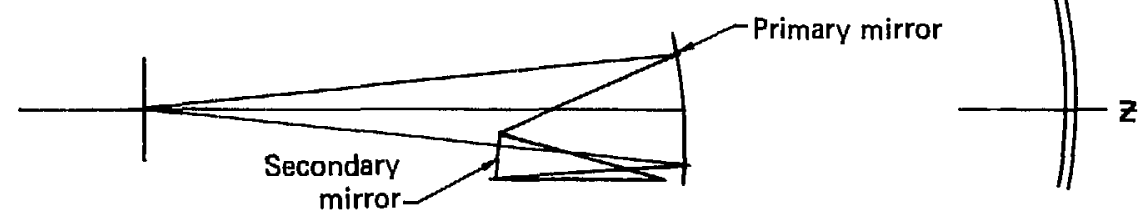

FIG. 13. MFTF objective mirror layout. (a) Overall view. (b) Off-axis mirror configuration. 
secondary mirror; to maintain throughput, an off-axis herschel system would be required. Relay optics would also be required to project the image out of the machine. The entire assembly would have to be steerable so it cuuld be autonatically aligned for each shot. With the space linitations imposed by the magnet, a machine wall, and the vacuum environment, this task would be difficult and expensive.

Lens systems, on the other hand, can accommodate a FoV substantially larger than the aperture. A lens system would have to be achromatic, because of the 200-nm spectral spread of the Thomson-scattered radiation (Fig. 8). The simplest objective system ( $\Gamma$ ig. 14) would be an achromat placeo about midway between the machine center and the wall, giving a magnification of about 1. An $\mathrm{f} / 5$ system would be required to give an aperture of 0.5 to $0.6 \mathrm{~m}$.

To examine the feasibility of such a system, the lens-design program ACos-V was used to calculate a basic Cooke triplet. We required that a point at the edge of the field of view produce a blur no more than $2 \mathrm{~mm}$ wide. The resulting lens design, with a mildly aspherical final surface, gives a blur $2 \mathrm{~mm}$ wide by $12 \mathrm{~mm}$ long. The length of the blur is caused by astigmatism; it is not a problem, because the $12-\mathrm{mm}$ focal line can be superimposed on the

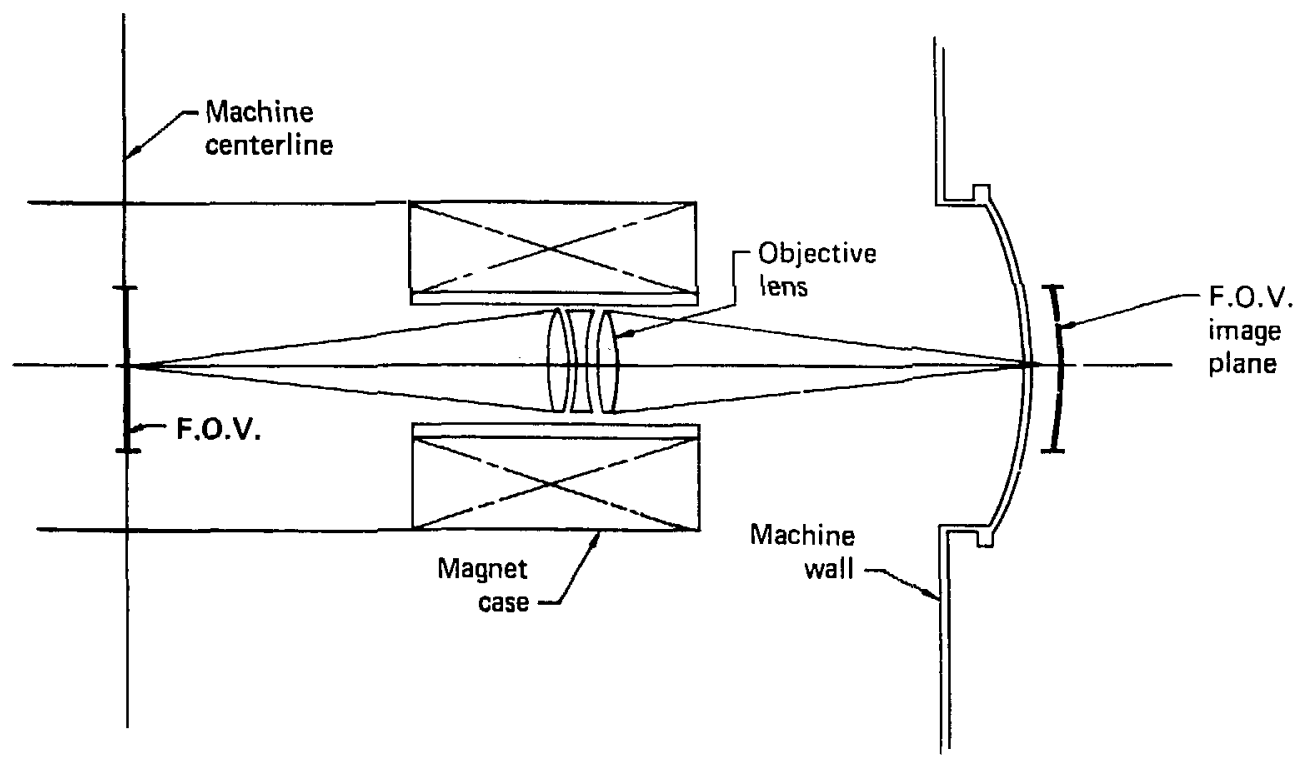

FIG. 14. MFTF objective lens layout. 
image of the laser. This use of a stigmatic image leads to some position uncertainty along the laser focal line but no broadening of the image, and therefore does not reduce the signal-to-noise ratio. There should be further design optimization, but this appears to be a feasible approach.

We required that the glasses be $\mathrm{CeO}_{2}$-doped for radiation resistance. The major cost item is the glass, estimated by the Optics Group at about $\$ 200,000$ for two units. (Because a custom melt and pouring is required, che cost is highly nonlinear with the number of units.) Fabrication and coating would bring the total cost to about $\$ 300,000$; it would take about a year to produce two units.

\section{RADIATION ENVIRONMENT}

Radiation loading on the optics will be a major problem. The objective, the largest and most expensive single component in the system, will also be the closest to the plasma and thus be subjected to the most radiation. The high-power laser components may have problems with the machine radiation and with the high-power laser beam itself.

The intensity of radiation from the plasma at the objective may be as high as $100 \mathrm{~W} / \mathrm{cm}^{2}$. This radiation is expected to be about $75 \%$ neutrals and $25 \%$ photons, the latter mostly UV and soft $x$-rays. In addition, fusion neutrons radiated by the plasma and by the walls wiil give the objective a 1 ifetime dose of about $10^{6}$ rad, primarily at $2.5 \mathrm{MeV}$.

This radiation environment will have two effects on optical components. Short-term thermal loading will distort components and may cause catastrophic failure. It may be necessary to use. a fused-silica heat shield to protect the objective. This problem requires further study. Cumulative, long-term absorption will cause the formation of color centers that reduce transmission and serve as damage centers for laser components.

Cumulative radiation damage to glass is a well-known phenomenon. Cerium-stabilized glasses that greatly reduce the problem have been developed (Fig. 15). Depths and rates of darkening differ somewhat with varying forms and energies of radiation, but visible effects occur at sufficiently low doses (about $10^{3} \mathrm{rad}$ ) that resistant glasses will have to be used. 

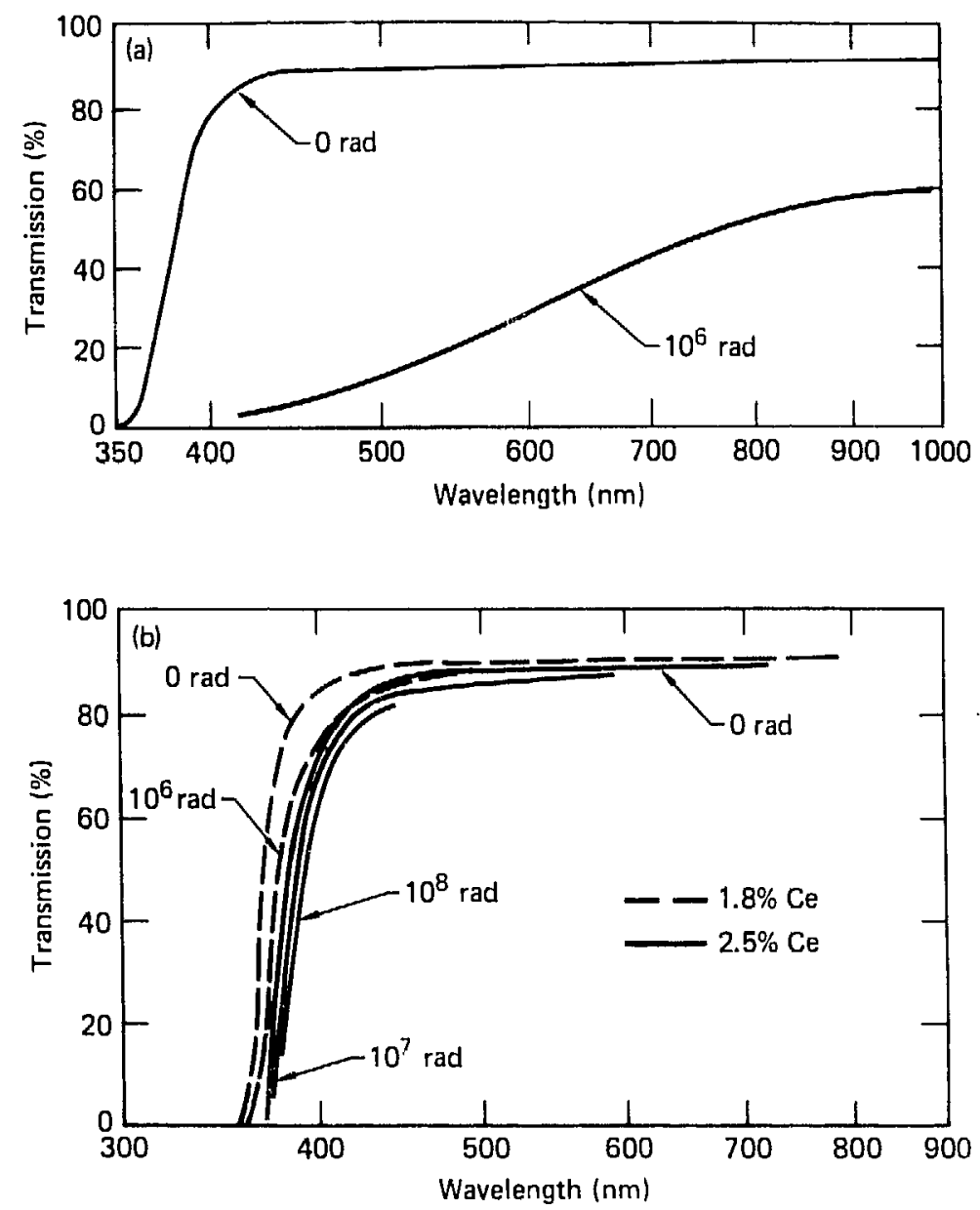

FIG. 15. Radiation darkening effects in (a) standard Bk-7 and (b) radiation-resistant (cerium-doped Bk-7) glass. (Courtesy: Scott Optical Glass, Inc.)

BEAM DUMP

The layout of observation points means that the laser beam will have to be aimable to different $z$ locations. If the beam is to be moved $0.5 \mathrm{~m}$ at the machine center, with a center of rotation (a mirror) at the machine wall, the beam motion at the opposite wall (the dump) will be $1 \mathrm{~m}$. This requires either a movable dump, a single slotted dump, multiple apertures, or multiple dumps. 
The TMX concept for suppressing light scatcered from both the input arm and the dump, shown schematically in Fig. 16, requires that all scattered laser light be intercepted by an absorbing surface. Apertures in the dump collimate this scatcered light so that it falls on the absurbing front aperture of the input arm. Thus the intensity $I$ of the light entering the machine is given by

$$
I=I_{0} S_{1} \Delta \&\left(R_{2}+S_{2}\right)
$$

where $I_{0} i$ is the intensity of the laser $\left(I_{0}=10^{20}\right.$ photons for a $10-\mathrm{J}$ pulse), $S_{1}$ and $S_{2}$ are the scattering coefficients of surfaces 1 and 2 in Fig. 16, respectively, and $k_{2}$ is the specular reflectivity of surface 2 . The solid angle $\Delta s$ is that subtended by the scattered light emerging from the dump. The total scattering from the dump surface, $\mathrm{s}_{1}$, is between $10^{-4}$ and $10^{-5}$ into $2 \pi \mathrm{sr}$. The combined reflection and scattering from the input-arm face, $R_{2}+s_{2}$, is about $2 \times 10^{-4}$. Thus the intensity into the machine (with the current design) is

$$
I=10^{-8} I_{0} \Delta \Omega
$$

If a slotted dump were used, most of the light scattered from the dump surface would emerge directly into the machine. Eliminating the input-arm term $\left(R_{2}+S_{2}\right)$, we obtain for the intensity

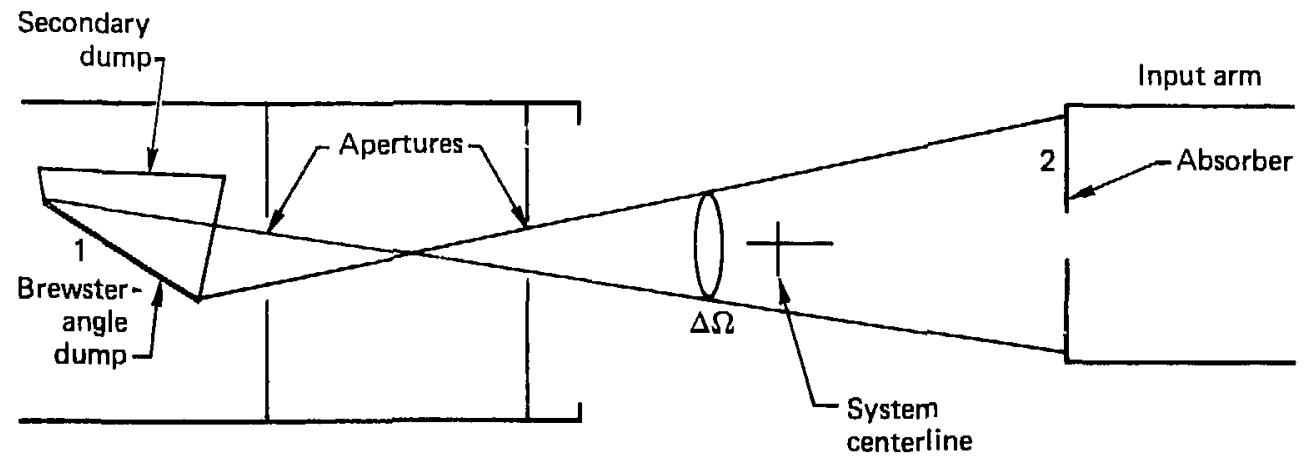

FIG. 16. TMX dump design. The dump constrains light scattered from dump surface (1) to fall on absorbing surface (2). 


$$
I \geq 10^{-5} I_{0} \Delta s_{\text {slot }}
$$

This is more than three orders of magnitude greater than the current design, and is unacceptable.

However, if MFTF is large enough and if the duration $\Gamma$ of the laser pulse is short enough (less than $10 \mathrm{~ns}$ ), time of flight might be used to help relax the dump requirements: If 1 ight from the scattering region reaches the objective before 1 ight bounced from a wall, a gated cetector can separate the two. The entrance aperture of the dump is generally the first surface to spill light into the machine. We require $2 \mathrm{CL}>\Gamma$, where $L$ is the distance from the foy of the viewing system to the dump surface. For a 10-ns pulse, $L$ must be greater than $2 \mathrm{~m}$, a reasonable requirement for MFTF; thus a slotted dump may be acceptable.

\section{POL'CHROMATORS}

A polychromator is the combination of a spectrometer and a multichanuel detector. The polychromators on $2 X \mathrm{IBB}^{19}$ and $\mathrm{TMX}^{20}$ use a commercial 0.5-m spectrometer coupled by fiber-optic light guides to a bank of ten photomultiplier tubes. Considerable work is in progress at several laboratories to make such polychromators more compact and efficient.

LaSalle and $P l a t z^{21}$ have developed a compact two-channel polychromator that has been used successfully on TFR. This system integrates wide spectral regions onto two PMT detectors. The two-channel approach yields good photon statistics and theoretically yields the same results as the ten-channel system on TMX. In the presence of spectral line emission, however, the two-channel system will give an erroneous result, while a noisy channel can be removed from a ten-channel system without significantly affecting the result.

Bretz et al.,$^{22}$ at the Princeton Plasma Physics Laboratory, are developing imaging detectors based on both Silicon Intensified Target (SIT) tubes and charge-coupled silicon detector arrays (CCDs). The concept of the imaging detector is thac all spatial sampling points are read out by the same detector. The system requires a complex and lossy fiber-optic image redistribution system to rearrange the sampling points along the laser beam to a format compatible with the spectrometer and detector.

There are several problems with the Princeton approach. The technique relies on a single detector, with no backup. The photocathode must be so 
large that only an S-20 photocathode can be used. The snortage of CCDs of suitable quality, and their poor ability to withstand integration into the intensifier, are severe limitations. Furthermore, cCDs would be susceptioie to neutron damage in MrTF.

The concept currently favored for MElE is to use separate, compact polychromators mounted directly in the image plane of the objective. Ihe detectors would be like those developed by Mous ${ }^{23}$ at Johns Hopkins for his 2a-channel vacum-UV analyzers. These detectors use shevron-plate intensifiers with an array of discrete-anode collectors; they are, in etfect, a Pil' array. The detecturs have 25-mm-diam active araa and are comnercially available with a choice of photocathodes, including semitransparent GaAs, which his high quantum efficiency. The anodes are approximately $2 \times 20$ m, so no image redistribution or compression is necassary. Thay are relatively

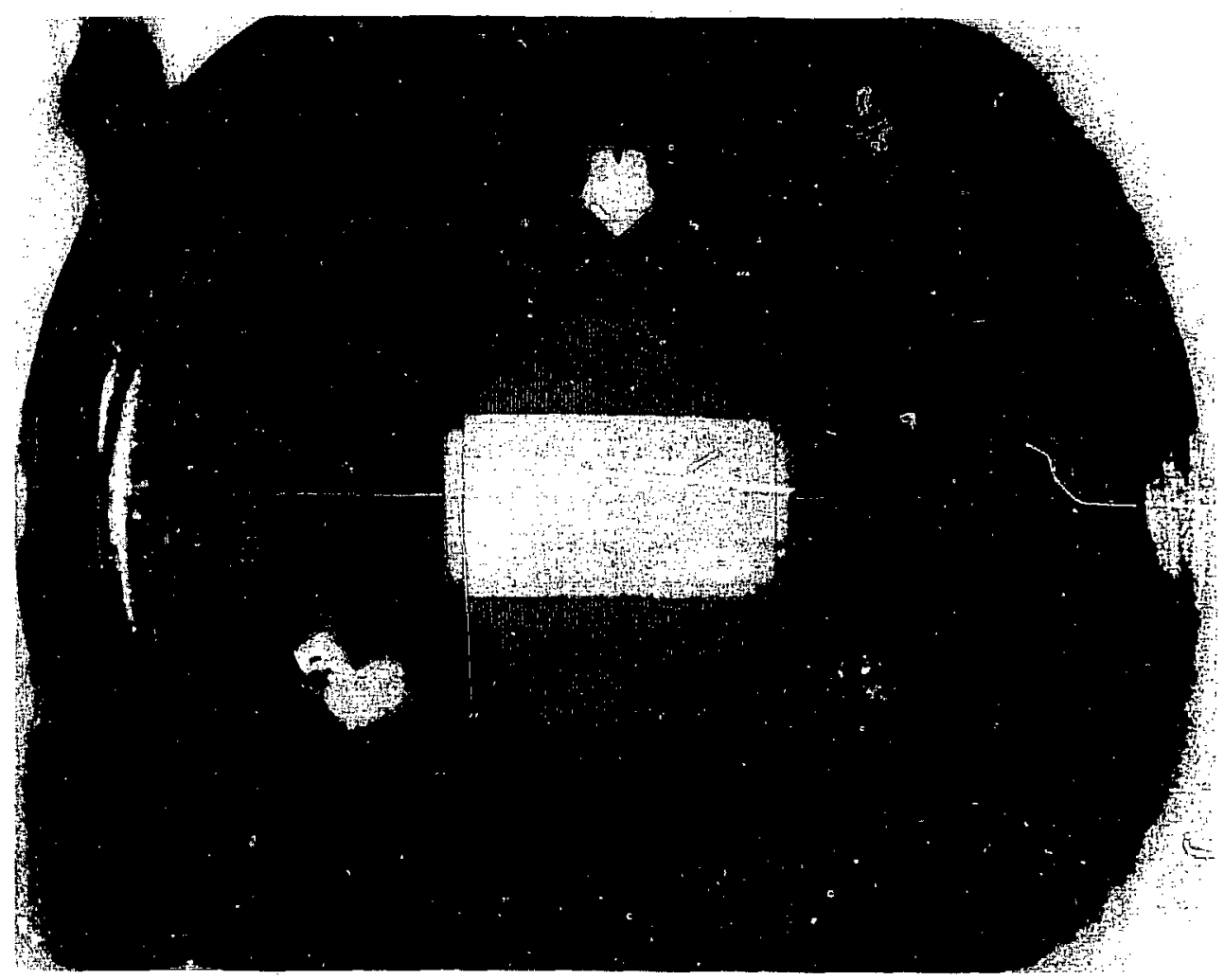

FIG. $17(\mathrm{a})$. A $1 \times 160-\mathrm{element}$ discrete-anode array. 




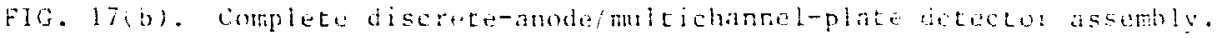

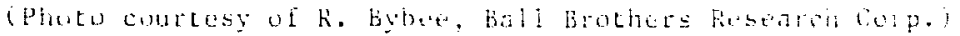

insensitive to magnetic fields and neutron damage, and are in use on lix."A spectrometer of focal lengch $0.2-0.3$ m would give enough divergence tor :he detector and would yield an extremely compact polychromacor. Similar detectors have been developed by tinothy et a 1.25 for aerospaco use (Fig. 17).

GONTINUING ISSUES

The issues $\mathrm{L}$ isced below are basic to meting the MFTF requirements. These issues become more important for MFTF-B, for which the physics requirements are more severe. 
LASER

The laser requirements, although not state-of-the-art individually, together define a frequency-doubled glass laser that has never been built. Quantel claims to be able to build such a laser in 6 to 12 months, but after such a laser is built and delivered about a year of debugging would probably be necessary to make it operational. Of course, manufacturer's claims lend to be speculative, particularly when there is no prior experience.

\section{BACKGROUND RADIATION}

Detailed spectral examination of the TMX background emission and of temperature and density scaling would provide a better understanding of the Thomson scattering constraints. This would also give the data necessary for masking spectral emissions other than $\mathrm{Da}$. A gated electronic spectrometer is required for the spectral examination, to differentiate between emissions characteristic of the startup, stable, and decay phases of a shot. More detailed examination of data from TMX and 2 XII would give additional scaling information.

HEAT-LOADING OF THE OBJECTIVE

The consequences of the $100 \mathrm{~W} / \mathrm{cm}^{2}$ radiative heat loading on the objective are not well understood. We must know if a heat shield is required, what damage it would suffer, and how long it would last. Answers to these questions require a finite-element thermal and stress analysis and further examination of the effects of particle radiation.

\section{CONCLUSIONS}

This report represents the end of development toward the MFTF parameters and a shift to the mcre stringent requirements of MFTE-B. Problems solved for MFTF arise again with MFTF-B. In particular, to measure $T_{e}$ up to about $10 \mathrm{keV}$ at ciensities below about $10^{13} \mathrm{~cm}^{-3}$ requires examination of forward-angle scattering, undoubled glass lasers, tradeoff of field of view for a more compact focal zone, auto-alignment, and background suppression 
techniques. The 30-s shot length on MFTF-B also allows consideraticn of continuous scattering and of synchronous detection methods. Although the Thomson scittering technology currently in use on TMX is not adequate, there appears to be sufficient upgrade capability to meet both MFTF and MFTF-B requirements.

\section{ACKNOWLEDGMENTS}

This study would not have been possible without fine support of many people throughout LLNL. In particular I thank Ron Goodman for theoretical consultations and experimental data, Rich Buck and Eric Frerking for computer support, and Lynn Seppala for lens design; I also thank MFT members Gil Leppelmeier, Stan Thomas, Jay Wiedwald, and Fred Coffield. 


\section{REFERENCES}

1. W. Löchte-Holtgreven, Plasma Diagnostics (North-Holland, Amsterdam, 1968), PP. 550-577.

2. T. P. Hughes, Plasmas and Laser Light (John Wiley and Sons, New York, 1975), Ch. 3 .

3. D. E. Evans, Plasma Physics; B. E. Keen, Ed. (Lectures from Culham Plasma Physics Summer Schools, Institute of Physics Conference Series Number 20, London, 1974), pP. 161-176.

4. A. M. Frank, Program TOMSCAT, Lawrence Livermore National Laboratory, Livermore, CA, UCID-18636 (1980).

5. F. Coffield, Requirements for Thomson Scattering, Lawrence Livermore National Laboratory, Livermore, CA, L Divisicn meeting minutes of March 28, 1980, 80-F-MN-3 (Apri1 2, 1980).

6. R. K. Goodman, Lawrence Livermore National Laboratory, Livermore, CA, private communication (March, 1980).

7. M. Mattioli and R. Papoular, Plasma Physics 17, 165-172 (1975).

8. J. Sheffield, Plasma Physics 14, 783-791 (1972).

9. Reference 2, T. P. Hughes, Plasmas and Laser Light (John Wiley, New York, 1975), Ch. 8 .

10. S. Von Goeler, W. Stodiek, H. Fishman, S. Grebenschikov, and E. Hinnov, Nuclear Fusion 15, 301-311 (1975).

11. M. Forrest, P. Jones, N. Peacock, R. Prentice, A. Selden, C. Walker, and S. Ward, "Design Study for Sp̣atial Scan Thomson Scattering System for JET," presented at Third APS Conference on Plasma Diagnostics, Los Angeles, CA, March 1980.

12. A. M. Frank, C. A. Anderson, and M. P. Paul, "Spectral Survey of TMX Center Cell Plasma," presented at APS Plasma Physics Meeting, San Diego, CA, November, 1980.

13. D. Deitrich and R. Fortner, Lawrence Livermore National Laboratory, Livermore, CA, private communication (1980).

14. S. Allen, Johns Hopkins University, Baltimore, MD, private communication (1980).

15. A. Peterson, B. Jacoby, and T. York, "Evaluating Frequency Doubled Glass Laser System for Thomson Scattering Plasma Diagnostics," presented at APS Plasma Physics meeting, San Diego, CA, Novembei, 1980. 
16. K. Hirsch, H. Salzmann, H. Röhr, and K. Steuer, "Quas i-Continuous Thomson Scattering Device for JET," presented at 3rd APS Conference on Plasma Diagnostics, Los Angeles, CA, March, 1980.

17. J. LaSalle and P. Platz, Applied Optics 18:24, 4124 (1979).

18. N. Bretz, Princeton Plasma Physics Laboratory, Princeton, NJ, private communication ( July, 1978).

19. T. C. Simonen, The 2XII Thomson Scattering System, Lawrence Livermore National Laboratory, Livermore, CA, UCRL-51493 (1973).

20. D. D. Lang, R. K. Goodman, S. L. Jenkins, J. A. Wilkerson, and J. L. Parkinson, Mechanical Design of the Thomson Scattering System for the TMX Fast Mirror Plug, Lawrence Livermore National Laboratory, Livermore, CA, UCRL-82912 (1979).

21. J. LaSalle and P. Platz, Opt. Commun. 17, 325 (1976).

22. N. Bretz, D. Dimmock, V. Foote, D. Johnson, D. Long, and E. Tolnas, Appl. Opt. 17, 192 (1978).

23. H. W. Mons, Johns Hopkins University, Baltimore, MD, private communication (1980).

24. T. Strand, W. Moos, and R. Hornady, "EUV Studies of Impurities on TMX," presented at Third APS Conference on High Temperature Plasma Diagnostics, Los Angeles, CA, March, 1980.

25. J. G. Timothy, G. H. Mount, and R. L. Bybee, "Detector Arrays for Photometric Measurements at Soft X-Ray, Ultraviolet, and Visible Wavelengths," Proc. SPIE 183, 169-181 (1979). 